\title{
SPITZER IRS SPECTRAL MAPPING OF THE TOOMRE SEQUENCE: SPATIAL VARIATIONS OF PAH, GAS, AND DUST PROPERTIES IN NEARBY MAJOR MERGERS
}

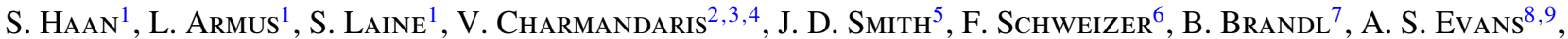 \\ J. A. Surace ${ }^{1}$, T. Diaz-Santos ${ }^{1,2}$, P. Beirão ${ }^{1}$, E. J. Murphy ${ }^{6}$, S. Stierwalt ${ }^{1}$, J. E. Hibbard ${ }^{8,9}$, M. Yun ${ }^{10}$, and T. H. Jarrett ${ }^{11}$ \\ ${ }^{1}$ Spitzer Science Center, California Institute of Technology, Pasadena, CA 91125, USA \\ ${ }^{2}$ Department of Physics and Institute of Theoretical and Computational Physics, University of Crete, GR-71003 Heraklion, Greece \\ ${ }^{3}$ IESL/Foundation for Research and Technology-Hellas, GR-71110, Heraklion, Greece \\ ${ }^{4}$ Chercheur Associé, Observatoire de Paris, F-75014 Paris, France \\ ${ }^{5}$ Ritter Astrophysical Observatory, University of Toledo, Toledo, OH 43606, USA \\ ${ }^{6}$ Observatories of the Carnegie Institution, 813 Santa Barbara Street, Pasadena, CA 91101, USA \\ ${ }^{7}$ Leiden Observatory, Leiden University, P.O. Box 9513, 2300 RA Leiden, The Netherlands \\ ${ }^{8}$ National Radio Astronomy Observatory, Charlottesville, VA 22903, USA \\ ${ }^{9}$ Department of Astronomy, University of Virginia, Charlottesville, VA 22904, USA \\ ${ }^{10}$ Astronomy Department, University of Massachusetts, 710 North Pleasant Street, Amherst, MA 01003, USA \\ ${ }^{11}$ Infrared Processing and Analysis Center, California Institute of Technology, Pasadena, CA 91125, USA \\ Received 2011 August 24; accepted 2011 October 11; published 2011 November 29
}

\begin{abstract}
We have mapped the key mid-IR diagnostics in eight major merger systems of the Toomre sequence (NGC 4676, NGC 7592, NGC 6621, NGC 2623, NGC 6240, NGC 520, NGC 3921, and NGC 7252) using the Spitzer Infrared Spectrograph. With these maps, we explore the variation of the ionized-gas, polycyclic aromatic hydrocarbon (PAH), and warm gas $\left(\mathrm{H}_{2}\right)$ properties across the sequence and within the galaxies. While the global PAH interband strength and ionized gas flux ratios ([Ne III]/[Ne II]) are similar to those of normal star-forming galaxies, the distribution of the spatially resolved PAH and fine structure line flux ratios is significantly different from one system to the other. Rather than a constant $\mathrm{H}_{2} / \mathrm{PAH}$ flux ratio, we find that the relation between the $\mathrm{H}_{2}$ and $\mathrm{PAH}$ fluxes is characterized by a power law with a roughly constant exponent $(0.61 \pm 0.05)$ over all merger components and spatial scales. While following the same power law on local scales, three galaxies have a factor of 10 larger integrated (i.e., global) $\mathrm{H}_{2} / \mathrm{PAH}$ flux ratio than the rest of the sample, even larger than what it is in most nearby active galactic nuclei. These findings suggest a common dominant excitation mechanism for $\mathrm{H}_{2}$ emission over a large range of global $\mathrm{H}_{2} / \mathrm{PAH}$ flux ratios in major mergers. Early-merger systems show a different distribution between the cold (CO $J=$ $1-0)$ and warm $\left(\mathrm{H}_{2}\right)$ molecular gas components, which is likely due to the merger interaction. Strong evidence for buried star formation in the overlap region of the merging galaxies is found in two merger systems (NGC 6621 and NGC 7592) as seen in the PAH, [Ne II], [Ne III], and warm gas line emission, but with no apparent corresponding CO $(J=1-0)$ emission. The minimum of the $11.3 / 7.7 \mu \mathrm{m}$ PAH interband strength ratio is typically located in the nuclei of galaxies, while the [Ne III/[Ne II] ratio increases with distance from the nucleus. Our findings also demonstrate that the variations of the physical conditions within a merger are much larger than any systematic trends along the Toomre sequence.
\end{abstract}

Key words: galaxies: evolution - galaxies: individual (NGC 4676, NGC 7592, NGC 6621, NGC 2623, NGC 6240, NGC 520, NGC 3921, NGC 7252) - galaxies: interactions - galaxies: starburst - ISM: molecules

Online-only material: color figures, figure sets

\section{INTRODUCTION}

Interactions and mergers are important drivers of galaxy evolution and are responsible for the formation of the most luminous infrared galaxies observed locally and many at high redshift. In particular, major mergers can completely disrupt the stellar and gaseous morphologies of the merging galaxies, transform spirals into massive ellipticals, and fuel both powerful starbursts and massive nuclear black holes (e.g., Barnes \& Hernquist 1992). In the most extreme cases, mergers can become ultraluminous infrared galaxies (ULIRGs), which are in many cases powered by massive starbursts with star formation rates sufficient to form the entire stellar population of an $\mathrm{L}^{*}$ galaxy in only a few dynamical times (Armus et al. 1987; Sanders et al. 1988; Heckman et al. 1990; Murphy et al. 1996). While most mergers do not produce ULIRGs, they invariably trigger largescale starbursts and lead to a large-scale redistribution of the progenitors' gaseous and stellar disks. At high redshift, mergers are responsible for the rapid evolution of the IR luminosity function seen in deep imaging surveys, and the formation of some of the most luminous galaxies detected at both UV and far-infrared wavelengths (e.g., Steidel et al. 1996; Blain et al. 2002; Chapman et al. 2005). At low redshifts, interactions and mergers provide to us a detailed picture of the most rapid and intense star formation known, and the generation and fueling of active nuclei.

The last decades have brought immense progress in the observations and modeling of both the stellar and the gaseous components of interacting galaxies. From the pioneering studies of Toomre \& Toomre (1972) and Schweizer (1982) through the work of Barnes \& Hernquist (1992) to the more recent work of Ferrarese et al. (2006), Côté et al. (2007), Kormendy et al. (2009), and Hopkins et al. (2009), it is now clear that interactions and mergers produce large-scale (tens of $\mathrm{kpc}$ ) stellar tidal tails, distorted and/or overlapping disks, central starbursts, and in some cases, stellar remnants that have the properties of elliptical galaxies. The gas, being dissipative, can rapidly form dense molecular disks, fueling both starbursts and an active 
nucleus (e.g., Scoville et al. 1991; Mihos \& Hernquist 1994; Charmandaris et al. 2002; Armus et al. 2004). Hubble Space Telescope (HST) observations of nuclei in merging galaxies have shown an increasing nuclear luminosity density in the optical/near-infrared light with advancing merger stage (Laine et al. 2003; Rossa et al. 2007; Veilleux et al. 2009; Haan et al. 2011), suggesting an increase of nuclear starburst activity. However, the fate of the dust in galactic interactions and mergers, and its direct relation to the gas and the young stars is much less well understood. For instance, a change in dust grain size, ionization stage, neutral to ionized gas fraction, or gas temperatures as function of merger stage could be expected. The dust responsible for re-radiating much of the UV light into the far-infrared can optically obscure the most energetic regions of star formation in interacting galaxies, which makes it challenging to explore the physical connection between the stars, gas, and dust in the nuclei, disks, and overlap regions where the brightest, most intense starbursts are triggered. While the correlation of enhanced far-infrared emission with galactic colors has been established (Soifer et al. 1984; Houck et al. 1985; Joseph \& Wright 1985; Sanders et al. 1986; Casoli et al. 1991; Surace et al. 2004), spatially resolved properties of the dust and gas within merging galaxies are still incomplete. To address the physical conditions in major mergers and excitation mechanisms such as shocks and starbursts, it is necessary to resolve the spatial variations of the warm dust, molecular gas, and ionized gas on subgalactic scales.

Here, we present Spitzer Infrared Spectrograph (IRS) spectral mapping observations of a sample of eight nearby, IR-bright galaxies that span the range from early through mid- to latestage major mergers. Our maps of the mid-IR features reveal the spatial variations of gas temperatures, masses, dust grain sizes, and the ionization state of the interstellar medium (ISM). In Section 2 we describe our sample, the observational setup, and the data reduction. The extracted spectra, mid-IR feature maps, dust, and warm gas properties (e.g., $\mathrm{H}_{2}$ temperature maps and masses) are presented in Section 3, compared to normal starforming galaxies, and discussed in the context of the physical properties of the ISM. Finally, we provide a brief overview of our conclusions in Section 4.

\section{OBSERVATIONS AND DATA REDUCTION}

\subsection{Sample}

By employing the Spitzer IRS in Spectral Mapping mode (Houck et al. 2004), we measure the gas and dust properties within a set of eight interacting/merging galaxies representing the early (NGC 4676, NGC 7592, NGC 6621-distinguishable parent disks and large tidal tails), middle (NGC 2623, NGC 6240, NGC 520-highly overlapping disk and/or double nuclei), and late (NGC 3921, NGC 7252 - single nucleus, elliptical-like light distribution) stages of the major merger process. In total, we obtained $44.9 \mathrm{hr}$ of Spitzer observing time in cycle 2 (PI: Lee Armus) to map these eight galaxies with the Short-Low (SL) and Long-Low (LL) modules of the IRS. All eight galaxies have Spitzer Infrared Array Camera (IRAC) and Multiband Imaging Photometer (MIPS) imaging, and are, except for NGC 6240, part of the classical "Toomre sequence" of merging galaxies (Toomre \& Toomre 1972; Toomre 1977). Note that the classical "Toomre sequence" lists galaxies only in rough order of completeness of the merger and is not meant to represent a precise order in time sequence of the merging process. An overview of the sample is given in Table 1. The
Table 1

Sample Overview

\begin{tabular}{lccrrrc}
\hline \hline Name & $\begin{array}{c}\text { R.A. } \\
(\mathrm{J} 2000)\end{array}$ & $\begin{array}{c}\text { Decl. } \\
(\mathrm{J} 2000)\end{array}$ & $\begin{array}{c}D \\
(\mathrm{Mpc})\end{array}$ & $\begin{array}{c}\log \left(L_{\mathrm{IR}} / L_{\odot}\right) \\
\log _{10}\left(L_{\odot}\right)\end{array}$ & T.S. & AGN Type \\
\hline NGC 520 & $01: 24: 35.1$ & $+03: 47: 33$ & 27.2 & $11.91^{1}$ & 5 & H II \\
NGC 2623 & $08: 38: 24.1$ & $+25: 45: 17$ & 80.6 & $11.54^{1}$ & 6 & LINER Sy2 \\
NGC 3921 & $11: 51: 06.9$ & $+55: 04: 43$ & 84.5 & $<10.35^{2}$ & 7 & H II \\
NGC 4676 & $12: 46: 10.7$ & $+30: 43: 38$ & 96.0 & $10.88^{3}$ & 1 & $\ldots$ \\
NGC 6240 & $16: 52: 58.9$ & $+02: 24: 03$ & 103.0 & $11.85^{1}$ & 4 & LINER Sy2 \\
NGC 6621 & $18: 12: 55.3$ & $+68: 21: 48$ & 85.2 & $11.23^{1}$ & 3 & H II \\
NGC 7252 & $22: 20: 44.7$ & $-24: 40: 42$ & 62.2 & $10.75^{3}$ & 8 & $\ldots$ \\
NGC 7592 & $23: 18: 22.2$ & $-04: 25: 01$ & 97.2 & $11.33^{1}$ & 2 & $\ldots$ \\
\hline
\end{tabular}

Notes. Summary of the properties of our sample of merger systems. Column 1: source name from NASA IPAC Extragalactic Database (NED); Column 2: right ascension from NED (J2000); Column 3: declination from NED (J2000); Column 4: the luminosity distance in Mpc (adopting $H_{0}=70 \mathrm{~km} \mathrm{~s}^{-1} \mathrm{Mpc}$ ), as provided by NED; Column 5 : the total infrared luminosity in $\log _{10}$ Solar units (1, Sanders et al. 2003; 2, Chitre \& Jog 2002; 3, Brandl et al. 2006); Column 6: the merger stage of the Toomre Sequence (from 1 to 8); Column 7: the AGN type derived from the optical and UV spectra, as recorded in NED.

galaxies are all nearby (mean distance of $79 \mathrm{Mpc}$ ), IR bright $\left(\log L_{\mathrm{IR}}\left[L_{\odot}\right]=10.3-11.9 .^{12}\right)$, and have a wealth of available ancillary data, from radio through X-rays (H I, CO, HST WFPC2 and Advanced Camera for Surveys (ACS), NICMOS, Very Large Array (VLA), Chandra). To illustrate the stellar distribution of these major mergers, we show in Figure 1 the IRAC $3.6 \mu \mathrm{m}$ images of the sample.

\subsection{IRS Observations and Data Reduction}

The SL and LL modules of the IRS are used to create threedimensional spectral data cubes over the central 1-2 $\operatorname{arcmin}^{2}$ in each system (corresponding to physical sizes of $\sim 8-28 \times$ $22-110 \mathrm{kpc})$ and over the entire wavelength interval $(5-38 \mu \mathrm{m})$. In all cases the maps are large enough to cover the entire main bodies (as defined by the optical and NIR images) and overlap regions. All SL and LL maps have half-slit width steps in the dispersion direction to avoid gaps and produce filled maps. The SL integrations are all $60 \mathrm{~s}$ with two cycles per position. The LL integrations are $120 \mathrm{~s}$ (one cycle), or $30 \mathrm{~s}$ (3-4 cycles) per position to maximize signal-to-noise ratio $(\mathrm{S} / \mathrm{N})$ and avoid saturation near the nuclei. The SL maps use sub-slit steps in the spatial direction to cover the intended area. We have designed the maps to cover the central regions of each galaxy, and at the same time provide multiple sky positions (from the non-primary slit) for background subtraction (see Figure 1). The IRS data reduction was performed using the interactive IDL software package CUBISM (Smith et al. 2007), which takes IRS Basic Calibrated Data (BCD) produced via spectral mapping mode, and constructs spectral data cubes suitable for detailed spatial and spectral analysis. CUBISM allows the user to construct twodimensional line and/or continuum images, and also extract one-dimensional (1D) spectra at any spatial position in the map (see Figure 1). Background spectra are constructed from our sky observations off the galaxies (when the primary slit is mapping the target, the secondary slit is looking at the sky next to the galaxy). The background is subtracted from the BCDs before the cubes are constructed. At a first step, line ([Ne II], [Ne III], $\mathrm{H}_{2}$ (S(0)-S(7)), [Ar II], [Si II]), and continuum (dust and polycyclic aromatic hydrocarbon, $\mathrm{PAH}$, feature) maps and 1D spectra are 12 This refers to estimates of the $8-1000 \mu \mathrm{m}$ infrared luminosity constructed
from IRAS flux densities as defined by Sanders et al. (2003). 

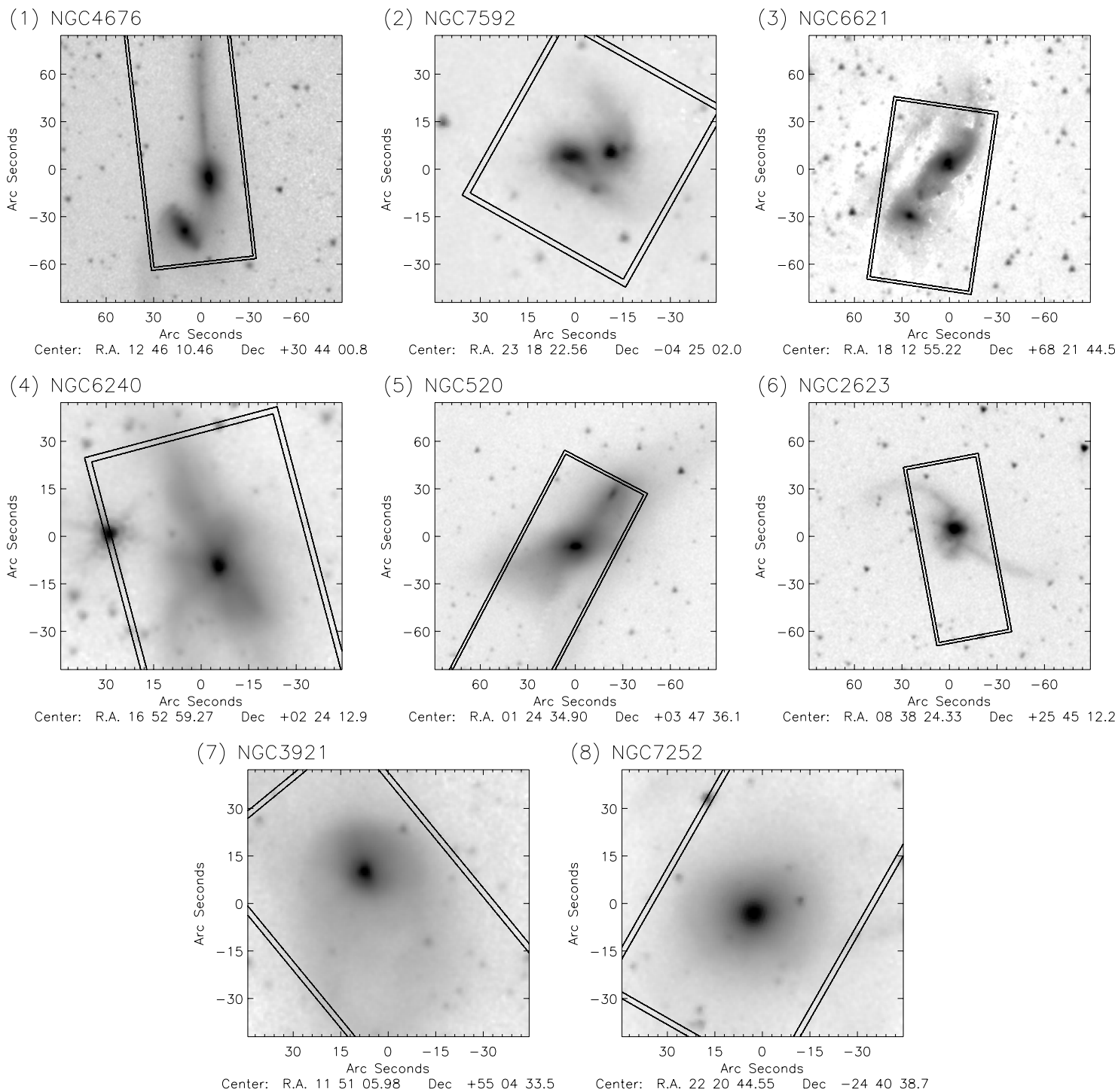

Figure 1. Spitzer IRAC $3.6 \mu \mathrm{m}$ images of our sample along the Toomre sequence (from 1-8, top-left to bottom-right). The field-of-view of the Spitzer IRS SL images is overlaid in rectangles and the figures are oriented such that north is up and east is left.

extracted and fit within CUBISM. These maps are later used as a benchmark for our maps created with PAHFIT (see Section 3.2).

\section{RESULTS AND DISCUSSION}

To investigate the properties of the warm gas and dust in our galaxies, we have studied the spatial distribution and global properties of the main mid-IR features, which are the primary PAH complex features at $6.2 \mu \mathrm{m}, 7.7 \mu \mathrm{m}, 11.3 \mu \mathrm{m}$, $12.7 \mu \mathrm{m}, 16.6 \mu \mathrm{m}$, and $17 \mu \mathrm{m}$, the primary diagnostics for the ionization state of the low-density gas ([Ne II] $12.81 \mu \mathrm{m}$, [Ne III] $15.55 \mu \mathrm{m},[\mathrm{Ar}$ II] $6.99 \mu \mathrm{m}$, [Si II] $34.82 \mu \mathrm{m}$, [O IV] $25.9 \mu \mathrm{m}$, and [Fe II] $25.99 \mu \mathrm{m}$ emission lines ${ }^{13}$ ), as well as the pure rotational lines of $\mathrm{H}_{2} \mathrm{~S}(0)$ to $\mathrm{S}(7)$ which probe the warm molecular gas (a detailed study of the $\mathrm{H}_{2}$ temperature and masses is elaborated in Section 3.3.2). Two different approaches are conducted. First, we study the global properties of the merger components, which has the advantage of high $\mathrm{S} / \mathrm{Ns}$ and allows us to compare our results with spatially integrated mid-IR properties of normal star-forming galaxies. Second, to

\footnotetext{
13 Note that the [O IV] $25.9 \mu \mathrm{m}$ and [Fe II] $25.99 \mu \mathrm{m}$ emission lines are blended in the LL data.
}

investigate in detail the distribution of the warm gas and dust, we map all mid-IR line features using a customized PAHFIT routine and also compare these maps with the cold gas distribution (using radio interferometer $\mathrm{CO}$ and $\mathrm{H}_{\mathrm{I}}$ emission lines) of these major mergers.

\subsection{D Spectra and Spectral Features}

To extract high $\mathrm{S} / \mathrm{N}$ spectra and line features, we have integrated the flux over those regions of each merger system that show significant emission in the spectral maps. These regions for each system (ranging from 2 to $20 \mathrm{kpc}$ ) are outlined on the IRS continuum maps in Figure 2. The continuum maps are constructed using the mean flux at 5.3-5.7 $\mu \mathrm{m}$ and 13.6-13.9 $\mu \mathrm{m}$, which are wavelength ranges with no significant emission line features. Spectra were extracted from the IRS SL $(5-15 \mu \mathrm{m})$ and LL $(15-37 \mu \mathrm{m})$ modules in spatially matched extraction regions using CUBISM. Each spectrum is composed of data from the four low-resolution orders of IRS: SL2 (5.25-7.6 $\mu \mathrm{m})$, SL1 (7.5-14.5 $\mu \mathrm{m})$, LL2 $(14.5-20.75 \mu \mathrm{m})$, and LL1 $(20.5-38.5 \mu \mathrm{m})$. Slight mismatches between order segments, resulting from small residual photometric and astrometric uncertainties, were addressed by fitting 
NGC 4676

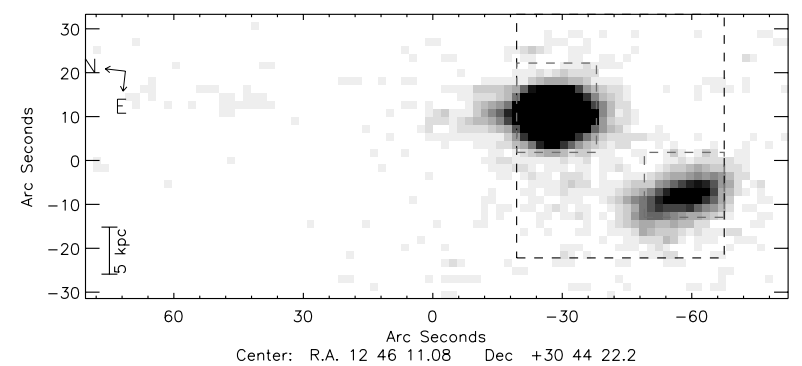

NGC 6621

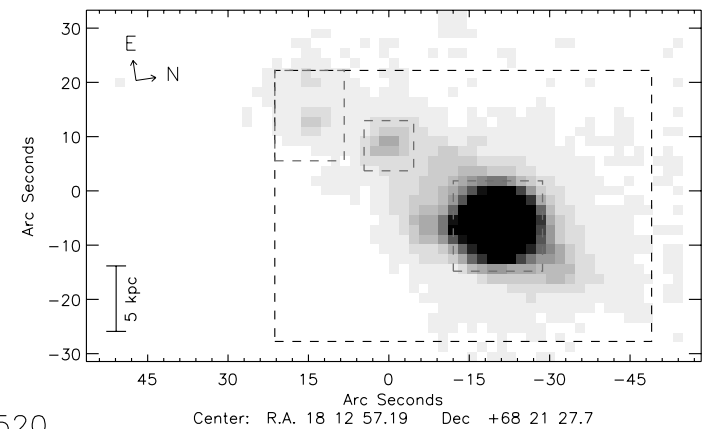

NGC 520

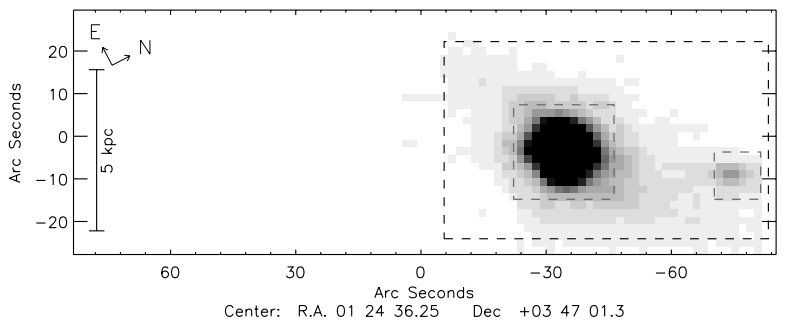

NGC 3921

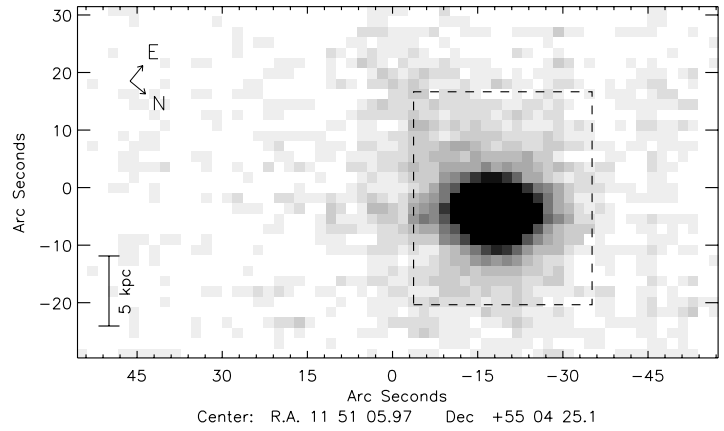

NGC 7592

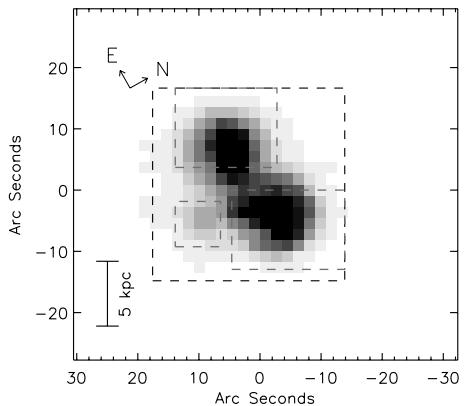

NGC 6240

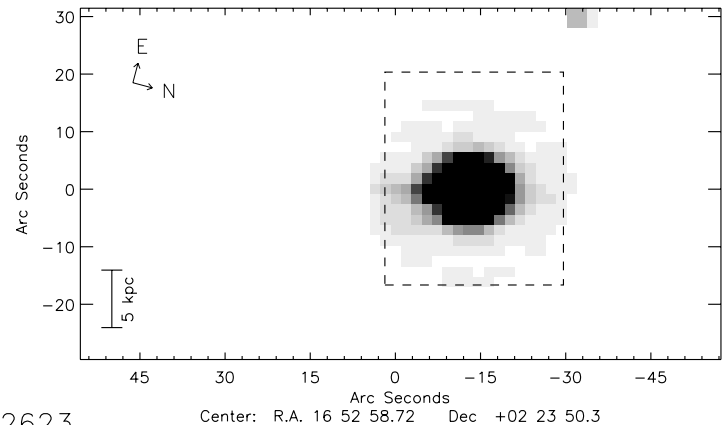

NGC 2623

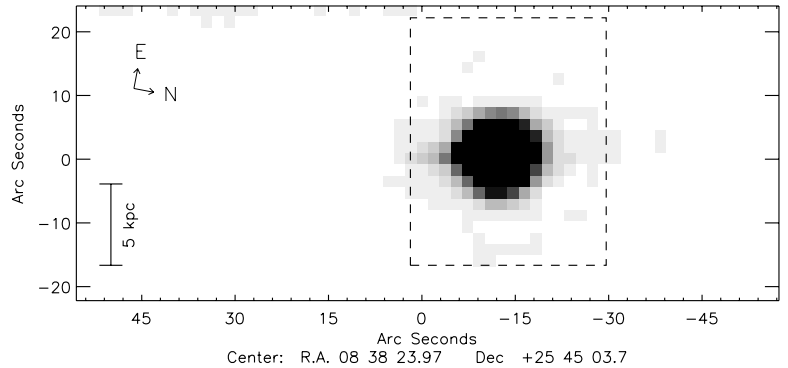

NGC 7252

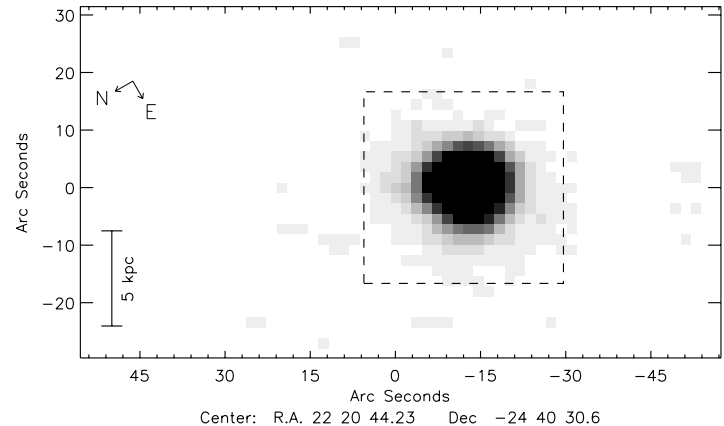

Figure 2. Mid-IR continuum maps of our sample (see Section 3.1 for more details). The dashed-line rectangles show the individual regions for which spectra have been extracted. North and east are marked by arrows in each frame and the bar corresponds to $5 \mathrm{kpc}$.

the continuum near the overlap region, then scaling SL2 to match SL1, LL1 to match LL2, and finally SL to match LL. The scaled spectra were then concatenated and averaged in the overlap region. Typical scaling adjustments were $\sim 10 \%$. To measure the line flux of the mid-IR features, we fit the combined SL/LL spectra using PAHFIT (Smith et al. 2007). This spectral fitting routine decomposes IRS spectra into broad PAH features, unresolved line emission, and grain continuum, with the main advantage to recover the full line flux of any blended features. Reliable redshifts of our objects are taken from NED. The best-fit solution of the observed spectrum is computed using a $\chi^{2}$ minimization method. The code returns the best-fit parameters for each of the components, including the line or feature fluxes and the optical depth of the silicate absorption feature at $9.7 \mu \mathrm{m}$.

PAHFIT provides several options to take dust extinction into account. We employed PAHFIT assuming a uniform foreground screen extinction (SCREEN model, i.e., a screen of dust between the galaxy and observer), dereddening the emitted line intensities with the extinction corrections estimated from the depth of the $9.7 \mu \mathrm{m}$ silicate absorption feature (Smith et al. 2007). The choice of a uniform screen model (over, e.g., a fully mixed dust geometry model or a galactic center extinction) is based on observations that have shown that a simple screen attenuation is able to account reasonably well for the diffuse ISM attenuation in normal and starburst galaxies (e.g., Smith et al. 
2007). The silicate extinction as given by the fitted $\tau$ at $9.7 \mu \mathrm{m}$ ranges from 0.08 to 1.4 (mean $\tau_{9.7}=0.7 \pm 0.5$, median: 0.6) for our sample, which is slightly larger than in normal galaxies (typically $\tau_{9.7}<0.2$; Smith et al. 2007) and starburst galaxies (median $\tau_{9.7}=0.23$; Brandl et al. 2006), but smaller than in LIRGs (median $\tau_{9.7}=1.4$; S. Stierwalt et al. 2011, in preparation) and ULIRGs (median $\tau_{9.7}=1.6$; Armus et al. 2007). To estimate the effect of dust extinction, we fixed in test-runs the extinction optical depth to zero and compared both results (extinction and extinction-free) using the $\mathrm{H}_{2}$ line flux at $9.66 \mu \mathrm{m}$. Since the $\mathrm{H}_{2}$ line at $9.66 \mu \mathrm{m}$ sits on top of the silicate absorption feature, we expect that this line is most affected by the extinction correction. We find that the models with no extinction have $\sim 20 \%$ lower $\mathrm{H}_{2}$ fluxes than the SCREEN models, indicating fairly small silicate absorption. Only for NGC 6240 and NGC 520 we find a three times smaller $\mathrm{H}_{2}$ flux with no-extinction than for the screen model, which is due to the large silicate absorption in these galaxies $\left(\tau_{9.7} \simeq 1.3\right)$. For a more thorough discussion of how PAHFIT determines quantities such as the uncertainty in the integrated line flux, line FWHM, line equivalent width, and the used mid-IR extinction law see Smith et al. (2007).

The combined SL/LL spectra and PAHFIT model are shown in Figure 3 for each region. The line flux and errors of the main mid-IR features are listed in Table 2. The 5-38 $\mu \mathrm{m}$ wavelength range contains many important diagnostic lines, such as unresolved atomic fine-structure lines of $\mathrm{Ar}, \mathrm{Ne}, \mathrm{O}, \mathrm{Si}$, $\mathrm{Fe}$, covering a large range in ionization potential. In addition to the fine-structure lines, numerous PAH emission features are easily detectable in our spectra; the fluxes of the strongest features at $6.2,7.7,11.3$, and the $17 \mu \mathrm{m}$ complex are listed in Table 2. We see a large range in global line intensities and $\mathrm{PAH}$ interband strengths for our galaxies as described in more detail in the following sections. Furthermore, the pure rotational lines of $\mathrm{H}_{2}[\mathrm{~S}(0)-\mathrm{S}(7)]$ are often very strong in starburst galaxies (Table 3) and are used in Section 3.3.2 to determine the temperature and mass of the (warm) molecular gas as listed in Table 4.

\subsection{Mapping Spectral Lines and PAH Emission}

In Figure 4, we present maps of the main mid-IR features for the eight major mergers in our sample. These continuumsubtracted maps were created using our own customized code with PAHFIT as core-algorithm. At first, we scale SL2 to match SL1, LL1 to match LL2, and finally SL to match LL by fitting the continuum near the overlap region (similar to the 1D spectra fitting procedure). The combined LL cube is rotated and interpolated in order to match the SL cube, conserving the flux. The scale factors are obtained using the entire range of pixels that match the LL and SL apertures, assuming a uniform scale factor rather than independent scale factors for each pixel. The aligned SL and LL cubes are concatenated on a common wavelength grid and averaged in the overlap wavelength regions. The maps in the combined SL/LL cubes $(5.3-38.5 \mu \mathrm{m})$ are smoothed by a 3 pixel $\times 3$ pixel box $\left(5^{\prime \prime} .55 \times 5^{\prime \prime} .55\right)$, conserving the flux, to increase the $\mathrm{S} / \mathrm{N}$ of the spectra. Then, for each pixel, the spectrum is extracted and PAHFIT is used to decompose it. Finally, we obtain maps of the properties of the fitted mid-IR features (i.e., integrated line flux, line FWHM, line equivalent width, the uncertainty in the line flux, the fit to the entire spectrum, and the fit to the continuum).
As a consistency check, we compared our line flux maps of the strongest line features with continuum-subtracted line flux maps created with CUBISM (see Section 2.2). The results show a very similar distribution of the strong line features, but CUBISM is less sensitive to faint extended emission in the outer disk. Overall, the main advantage of fitting each pixel is that faint local variations can be taken into account that would not be visible by subtracting only the continuum averaged over all the pixels. Another important advantage of this method over CUBISM is to fit the full features before creating the maps, which would otherwise tend to lose wings of the broad PAH features and having difficulties to deblend the PAH features from each other, and other continuum features (e.g., the silicate absorption). In some mid-IR feature maps (Figure 4) we find faint rings which are artifacts of the continuum subtraction (also present in CUBISM derived maps, although in a smaller degree).

\subsubsection{Continuum Fluxes}

To characterize the basic properties of the spectral continuum we have mapped the flux densities for three rest-frame wavelength ranges: $5.3-5.7,14.75-15.25$, and $29.5-30.5 \mu \mathrm{m}$. These wavelengths were originally chosen to cover a large baseline in wavelength while being least affected by PAH emission features, silicate absorption, or strong emission lines. The flux densities derived for $5.5,15$, and $30 \mu \mathrm{m}$ are the median values in the above wavelength ranges, respectively. Although the continuum fit derived with PAHFIT is not affected by features, we have chosen these wavelength ranges to be consistent with CUBISM-derived maps and previous IRS studies for comparison. All of our galaxies show a decrease of the $30-15 \mu \mathrm{m}$ continuum flux ratio toward their center. In general, also a smaller $15 / 5 \mu \mathrm{m}$ ratio in the galaxies' center is found, but in some cases we find strong spatial deviations from the azimuthal averaged profile (NGC 2623, NGC 4676, NGC 520, and NGC 3921).

The spatially integrated $30 / 15 \mu \mathrm{m}$ flux ratio of our sample ranges from 5 (NGC 6621, NGC 3921, NGC 7252) to 16 (NGC 2623) with a mean value of 7.3, which is similar to measurements of starburst galaxies (mean $30 / 15 \mu \mathrm{m}$ flux ratio of 8.56; Brandl et al. 2006). The same behavior is seen for the spatially integrated $15 / 5.5 \mu \mathrm{m}$ flux ratio which ranges from 1.5 (NGC 6621, NGC 3921) to 6.3 (NGC 2623) with a mean value of 3.4 (3.8 for starburst galaxies; Brandl et al. 2006). Two merger components in our sample show significantly smaller ratios than the ratios averaged over the entire merger system: NGC 4676 South $(30 / 15 \mu \mathrm{m}=2.2,15 / 5.5 \mu \mathrm{m}=0.8)$ and NGC 6621 South $(30 / 15 \mu \mathrm{m}=2.0,15 / 5.5 \mu \mathrm{m}=0.5)$.

\subsubsection{Polycyclic Aromatic Hydrocarbons}

PAHs are thought to be responsible for a series of broad emission features that dominate the mid-infrared spectra of starbursts (see, e.g., Peeters et al. 2004). They are observed in a diverse range of sources, with their strongest emission originating in photodissociation regions (PDRs), the interfaces between $\mathrm{H}$ II regions and molecular clouds. The relative strength of the different PAH bands is expected to vary with the size and the ionization state of the PAH molecule (Draine \& $\mathrm{Li}$ 2001; Smith et al. 2007). The $6.2 \mu \mathrm{m}$ and $7.7 \mu \mathrm{m}$ PAH bands dominate the emission of ionized PAH, whereas the $11.3 \mu \mathrm{m}$ PAH emission is more intense for neutral PAHs. Variations of PAH features in nearby galaxies have been studied recently by Galliano et al. (2008), Alonso-Herrero et al. (2010), PereiraSantaella et al. (2010), and Díaz-Santos et al. (2010a, 2010b, 

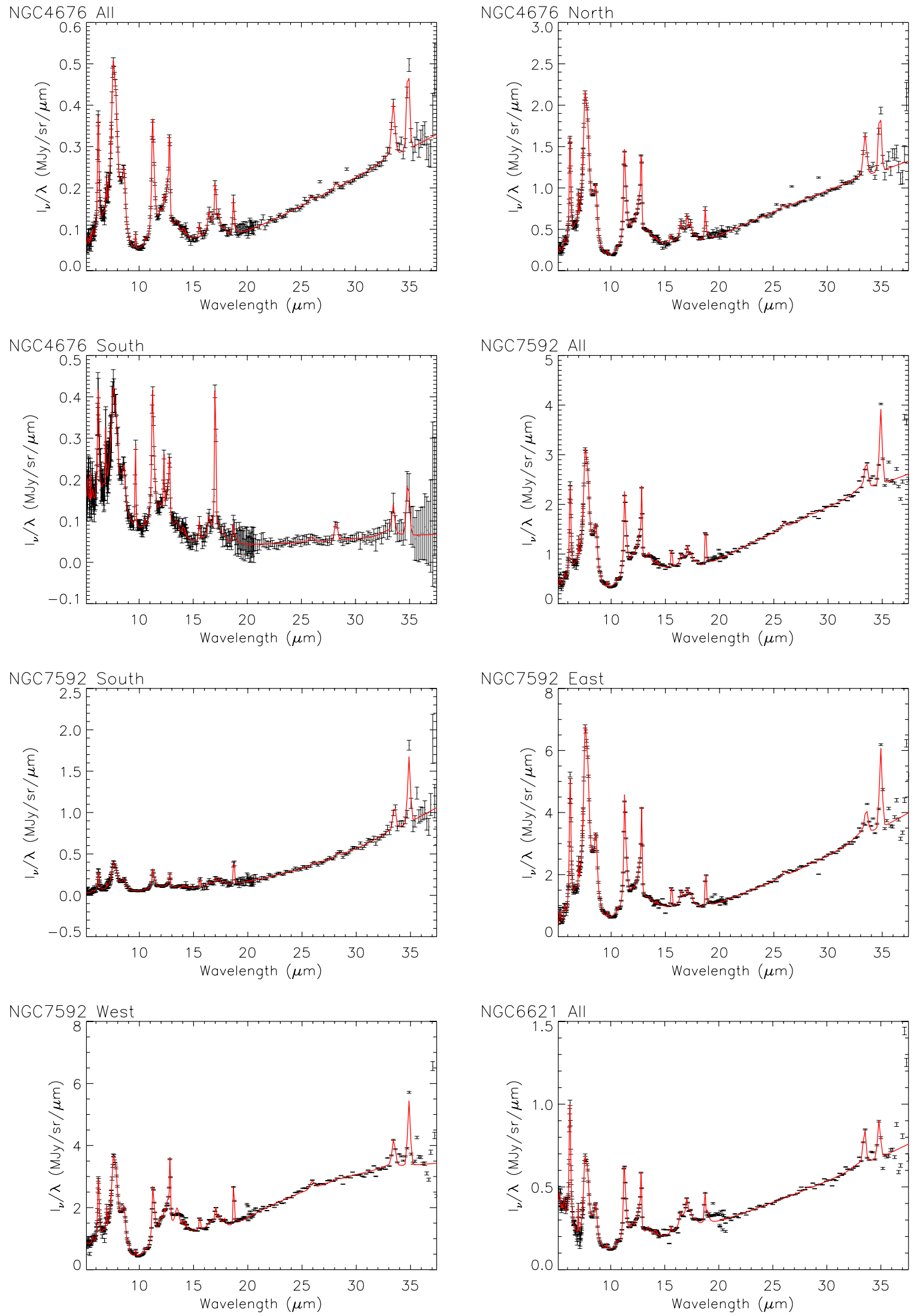

Figure 3. IRS spectra (black error bars) fitted with PAHFIT (red line). The size and central coordinates of the individual regions are given in Table 2. (A color version of this figure is available in the online journal.) 

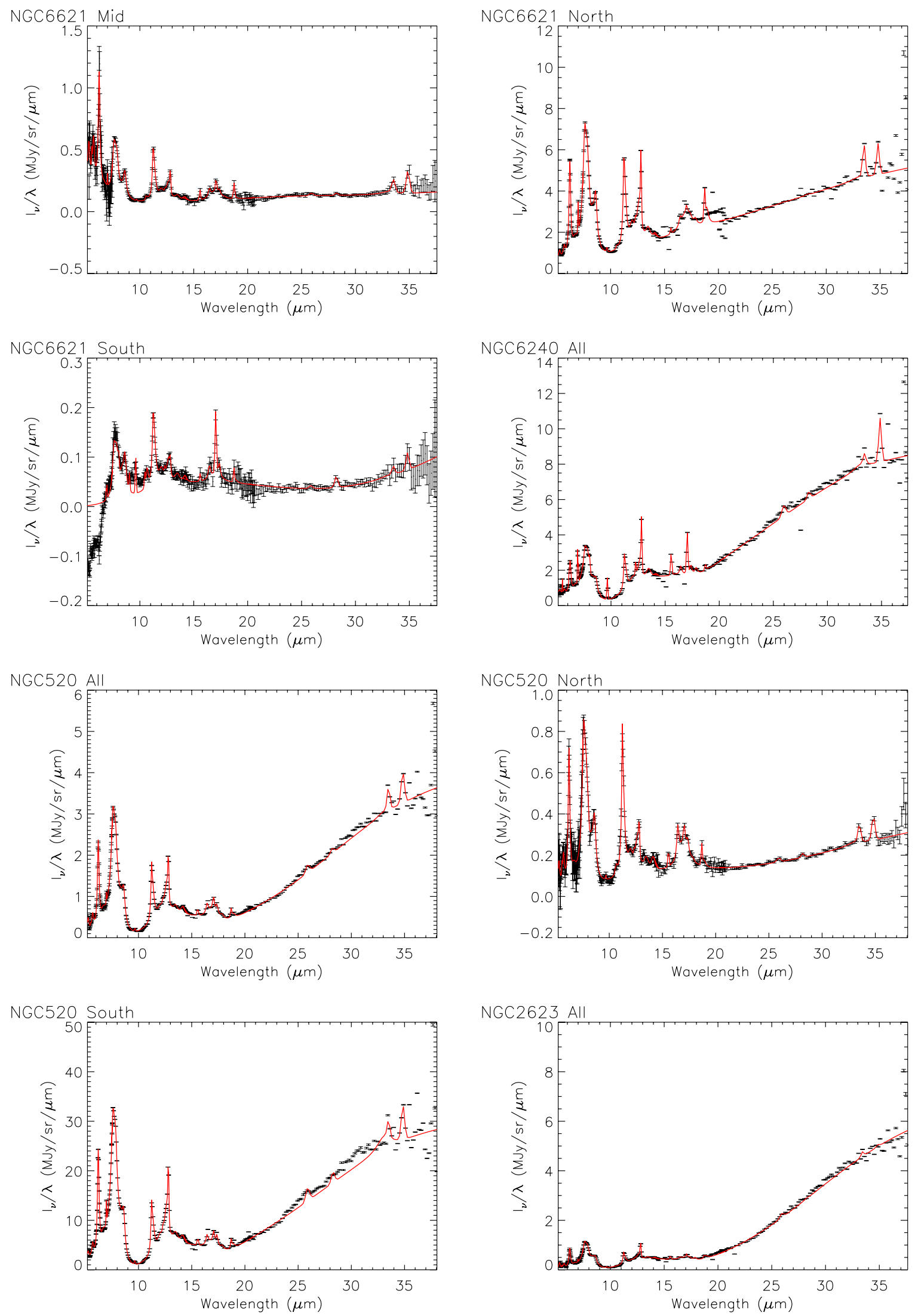

Figure 3. (Continued) 

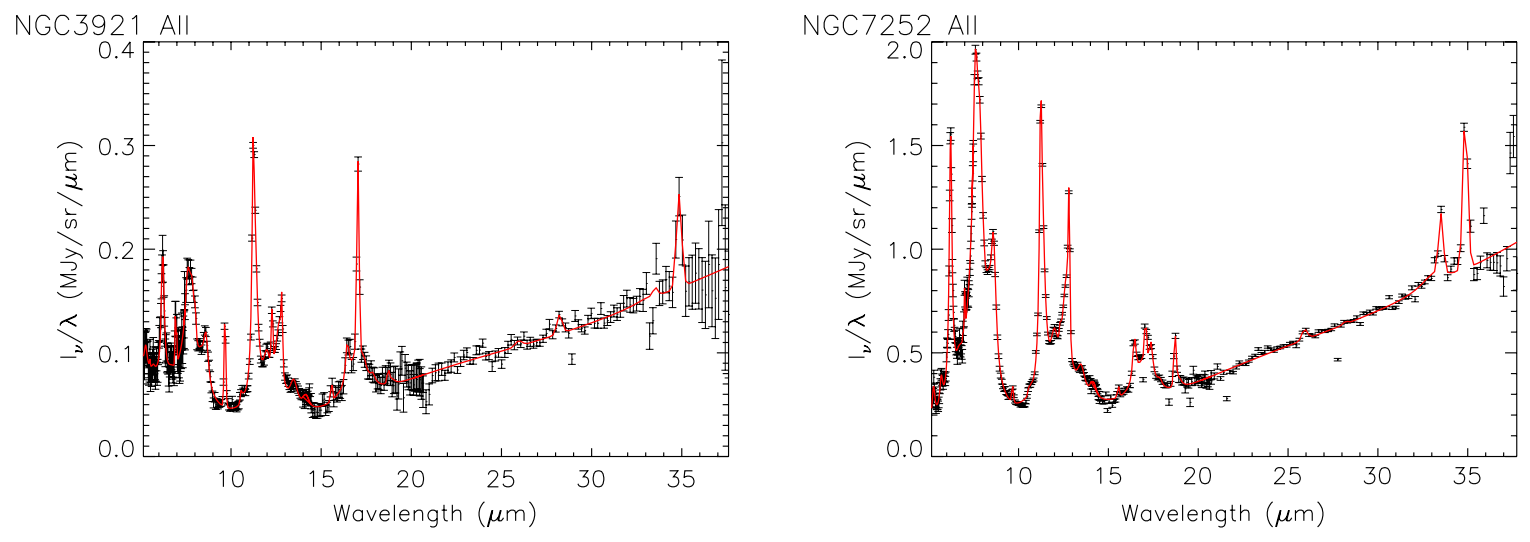

Figure 3. (Continued)

2011), including the effects of the radiation field hardness and intensity, the size distribution of the PAH carriers, extinction effects, and the dependency on the age of the stellar population.

Our spectra in Figure 3 show that the spectral shape is usually dominated by strong emission features from PAHs. Here, we concentrate our analysis on several of the strongest PAH features at $6.2 \mu \mathrm{m}, 7.7 \mu \mathrm{m}$ (which is three blended sub-features centered at 7.42, 7.60, and $7.85 \mu \mathrm{m}$ ), $11.3 \mu \mathrm{m}$ (two blended sub-features at 11.23 and $11.33 \mu \mathrm{m}$ ), and around $17 \mu \mathrm{m}$ (two blended PAH sub-features centered at 16.4 and $17.1 \mu \mathrm{m}$ ). Other PAH features can be seen in the spectra (e.g., at 8.6 or $12.7 \mu \mathrm{m}$ ), but they have in general lower $\mathrm{S} / \mathrm{N}$ or are blended with other features. The fluxes of the PAH bands at 6.2, 7.7, and $11.3 \mu \mathrm{m}$ are all affected by dust extinction, but the $11.3 \mu \mathrm{m}$ is likely more affected by the $9.7 \mu \mathrm{m}$ silicate feature than the $6.2 \mu \mathrm{m}$ feature. The $7.7 \mu \mathrm{m}$ PAH feature is affected by extinction to a similar level as the $6.2 \mu \mathrm{m}$ feature (Draine \& Li 2001; Beirão et al. 2008).

In Figures 5 and 6, we present the PAH $6.2 / 7.7 \mu \mathrm{m}$ and $7.7 / 11.3 \mu \mathrm{m}$ ratio maps for the Toomre sequence. For most of our galaxies the PAH feature at $11.3 \mu \mathrm{m}$ is more extended than the PAH feature at $7.7 \mu \mathrm{m}$ (factor of $\sim 2$, ranging from 1.3 to 3.3 ), equivalent to an increase of the PAH $7.7 / 11.3 \mu \mathrm{m}$ ratio toward the center, which is in agreement with previous studies (Galliano et al. 2008; Pereira-Santaella et al. 2010; Díaz-Santos et al. 2011). This is expected, since the $7.7 \mu \mathrm{m}$ PAH feature is related to the ionized PAHs (which are more likely located in the central starburst region) rather than neutral PAHs, which are traced by PAH $11.3 \mu \mathrm{m}$ and are located more likely in diffuse regions throughout the galaxies. However, for three galaxies (NGC 4676 North, NGC 6240, and NGC 2623) we find the opposite behavior, namely, a decreasing PAH 7.7/11.3 $\mu \mathrm{m}$ ratio toward the center, which has been also found in the center of weak active galactic nucleus (AGN) host galaxies (Smith et al. 2007; O'Dowd et al. 2009; Diamond-Stanic \& Rieke 2010; Sales et al. 2010, e.g.,) and suggested to be attributed to the selective destruction of the $7.7 \mu \mathrm{m}$ PAH molecule with the increasing hardness of the radiation field. This picture is supported by our observations since two of the galaxies mentioned above (NGC 6240 and NGC 2623) are characterized as low luminosity AGN hosts (see Table 1), whereas none of the rest of the sample seems to host an AGN. We find for most of our galaxies a decreasing PAH 6.2/7.7 $\mu \mathrm{m}$ ratio toward the center (typically a factor of two). This is rather surprising since both bands, $6.2 \mu \mathrm{m}$ and $7.7 \mu \mathrm{m}$, are related to ionized PAHs and have shown only very small variations in previous studies (Galliano et al. 2008; Pereira-Santaella et al. 2010). Since the PAH 6.2 and $7.7 \mu \mathrm{m}$ ratio decreases toward larger grain sizes (larger number of PAH carbon atoms), an intuitive explanation is that the PAHs with larger grain sizes are more likely located in the outskirts rather than in the center, which is in agreement with models of PAHs in PDRs (Draine \& Li 2001). A possible correlation between PAH grain size and ionization state is discussed in the next section.

\subsubsection{PAH Interband Strengths and Ionization}

To characterize the distribution and population of the PAH grain sizes and ionization states for each merger system, we present in Figure 7 the variation of interband strength ratios of the PAH features and [Ne III]/[Ne II] ratios (integrated over apertures of $3 \times 3$ pixels or $5^{\prime \prime} .6 \times 5^{\prime \prime} .6$ ) where significant emission is present, and compared to PAH models by Draine \& Li (2001). In general, neutral PAHs have large ratios of PAH 11.3/7.7 $\mu \mathrm{m}$, while PAH ions have much smaller values. For both neutrals and ions, there is a regular progression of PAH 6.2/7.7 to smaller values with increasing number of PAH carbon atoms (Draine $\&$ Li 2001).

We find a large variety of PAH distributions, ranging from ionized to neutral PAHs over a large range in grain sizes (from a few ten to thousands of carbon atoms). For instance, NGC 4676 (primarily ionized PAHs) and NGC 520 (primarily neutral PAHs) show a very concentrated distribution in the PAH interband strength ratios, while, e.g., NGC 6621 (ionized and neutral PAHs) and NGC 3921 (primarily neutral PAHs, including the nucleus) exhibit more widespread $\mathrm{PAH}$ populations. We find no significant correlation between PAH grain size (PAH $6.2 / 7.7 \mu \mathrm{m}$ ) and ionization state (PAH 11.3/7.7 $\mu \mathrm{m})$. Instead, we find a large variety of $\mathrm{PAH}$ grain sizes for a given charge state, e.g., for NGC 7592, NGC 6240, and NGC 7252. NGC 3921 is mainly populated by neutral PAHs. For most of our galaxies, the central apertures (center of galaxies) have low PAH 11.3/7.7 $\mu \mathrm{m}$ ratios, representing typically the most ionized $\mathrm{PAH}$ population for each galaxy, as one would expect from a central starburst or a low-luminosity AGN. This finding is in agreement with other recent studies of PAH populations in ULIRGs (Díaz-Santos et al. 2011) which show that the PAH $11.3 \mu \mathrm{m}$ is spatially more extended than the PAH $7.7 \mu \mathrm{m}$ feature.

\subsubsection{The [Ne III]/[Ne II] Line Ratios: Probing the Hardness of the Radiation Field}

In general the ratio of the $12.81 \mu \mathrm{m}$ [Ne II] and $15.55 \mu \mathrm{m}$ [Ne III] lines provides a diagnostic of ionization that is relatively insensitive to extinction, gas-phase abundances, and gas temperature (being low excitation lines in warm gas). Since the two ionization potentials of $\mathrm{Ne}$ are $21.6 \mathrm{eV}$ [Ne II] and $41.0 \mathrm{eV}$ 
Table 2

Mid-IR Emission Line and PAH Features

\begin{tabular}{|c|c|c|c|c|c|c|c|c|c|}
\hline Name & $\begin{array}{l}\text { R.A. } \\
\text { (J2000) }\end{array}$ & $\begin{array}{l}\text { Decl. } \\
(\text { J2000) }\end{array}$ & $\begin{array}{l}\text { Region Size } \\
\left(10^{-9} \text { sterad }\right)\end{array}$ & $\begin{array}{c}\text { PAH } 6.2 \mu \mathrm{m} \\
\left(10^{-9} \mathrm{~W} \mathrm{~m}^{-2} \mathrm{sr}^{-1}\right)\end{array}$ & $\begin{array}{c}\text { PAH } 7.7 \mu \mathrm{m} \\
\left(10^{-9} \mathrm{~W} \mathrm{~m}^{-2} \mathrm{sr}^{-1}\right)\end{array}$ & $\begin{array}{c}\text { PAH } 11.3 \mu \mathrm{m} \\
\left(10^{-9} \mathrm{~W} \mathrm{~m}^{-2} \mathrm{sr}^{-1}\right)\end{array}$ & $\begin{array}{c}\text { PAH } 17 \mu \mathrm{m} \\
\left(10^{-9} \mathrm{~W} \mathrm{~m}^{-2} \mathrm{sr}^{-1}\right)\end{array}$ & $\begin{array}{c}{[\mathrm{Ne} \text { II] } 12.81 \mu \mathrm{m}} \\
\left(10^{-9} \mathrm{~W} \mathrm{~m}^{-2} \mathrm{sr}^{-1}\right)\end{array}$ & $\begin{array}{c}{[\mathrm{Ne} \text { III] } 15.55 \mu \mathrm{m}} \\
\left(10^{-9} \mathrm{~W} \mathrm{~m}^{-2} \mathrm{sr}^{-1}\right)\end{array}$ \\
\hline NGC 520 System & 01:24:35.10 & $+03: 47: 33.0$ & 75.5 & $389.5 \pm 2.4$ & $1427.7 \pm 7.1$ & $304.9 \pm 1.5$ & $207.3 \pm 1.3$ & $34.5 \pm 0.2$ & $6.1 \pm 0.1$ \\
\hline NGC 520 North & $01: 24: 33.36$ & $+03: 48: 02.5$ & 8.8 & $90.8 \pm 4.8$ & $264.5 \pm 14.2$ & $77.2 \pm 1.4$ & $59.3 \pm 2.0$ & $4.1 \pm 0.3$ & $2.2 \pm 0.2$ \\
\hline NGC 520 South & 01:24:34.90 & $+03: 47: 29.5$ & 6.0 & $4171.2 \pm 10.0$ & $16167.2 \pm 28.0$ & $2677.9 \pm 5.8$ & $1670.1 \pm 6.8$ & $407.8 \pm 0.7$ & $58.2 \pm 0.6$ \\
\hline NGC 2623 & 08:38:24.11 & $+25: 45: 16.4$ & 33.5 & $145.3 \pm 2.5$ & $493.8 \pm 6.4$ & $99.9 \pm 1.1$ & $95.7 \pm 1.7$ & $17.6 \pm 0.2$ & $4.8 \pm 0.2$ \\
\hline NGC 3921 & $11: 51: 06.86$ & $+55: 04: 43.6$ & 46.4 & $16.9 \pm 1.4$ & $48.4 \pm 4.2$ & $31.7 \pm 1.5$ & $18.1 \pm 1.8$ & $1.7 \pm 0.1$ & $0.5 \pm 0.2$ \\
\hline NGC 4676 System & $12: 46: 10.70$ & $+30: 43: 38.0$ & 69.8 & $45.9 \pm 1.4$ & $187.5 \pm 5.0$ & $41.5 \pm 1.5$ & $23.6 \pm 2.1$ & $5.2 \pm 0.1$ & $1.0 \pm 0.2$ \\
\hline NGC 4676 North & $12: 46: 10.09$ & $+30: 43: 55.1$ & 11.2 & $216.0 \pm 3.5$ & $830.9 \pm 7.7$ & $185.3 \pm 3.0$ & $104.1 \pm 4.9$ & $23.7 \pm 0.2$ & $3.2 \pm 0.5$ \\
\hline NGC 4676 South & $12: 46: 11.28$ & $+30: 43: 21.5$ & 9.7 & $40.2 \pm 3.4$ & $136.6 \pm 10.1$ & $38.4 \pm 1.0$ & $20.7 \pm 2.8$ & $3.5 \pm 0.3$ & $1.4 \pm 0.4$ \\
\hline NGC 6240 & $16: 52: 58.92$ & $+02: 24: 03.2$ & 32.3 & $314.0 \pm 2.5$ & $1490.8 \pm 6.2$ & $369.1 \pm 1.0$ & $147.2 \pm 1.4$ & $88.9 \pm 0.2$ & $44.2 \pm 0.2$ \\
\hline NGC 6621 System & 18:12:55.30 & $+68: 21: 48.0$ & 340.0 & $108.3 \pm 1.1$ & $164.4 \pm 0.9$ & $58.6 \pm 0.2$ & $46.8 \pm 0.4$ & $8.1 \pm 0.1$ & $1.0 \pm 0.1$ \\
\hline NGC 6621 North & $18: 12: 55.49$ & $+68: 21: 47.8$ & 34.4 & $630.6 \pm 2.8$ & $2380.7 \pm 8.5$ & $535.6 \pm 1.0$ & $368.8 \pm 1.6$ & $92.0 \pm 0.3$ & $7.7 \pm 0.2$ \\
\hline NGC 6621 South & 18:12:59.78 & $+68: 21: 14.8$ & 51.5 & & $38.0 \pm 1.8$ & $19.9 \pm 0.5$ & $9.0 \pm 1.4$ & $0.8 \pm 0.1$ & $0.4 \pm 0.2$ \\
\hline NGC 6621 Middle & $18: 12: 58.58$ & $+68: 21: 28.9$ & 10.5 & $135.1 \pm 7.0$ & $142.9 \pm 3.4$ & $55.9 \pm 1.8$ & $34.3 \pm 2.3$ & $4.5 \pm 0.2$ & $2.2 \pm 0.3$ \\
\hline NGC 7252 & $22: 20: 44.77$ & $-24: 40: 41.7$ & 34.2 & $188.6 \pm 2.6$ & $660.6 \pm 8.8$ & $191.8 \pm 3.1$ & $92.5 \pm 2.9$ & $18.4 \pm 0.2$ & $1.4 \pm 0.2$ \\
\hline NGC 7592 System & 23:18:22.20 & $-04: 25: 01.0$ & 27.6 & $321.5 \pm 3.4$ & $1146.4 \pm 13.0$ & $250.9 \pm 1.9$ & $127.3 \pm 2.0$ & $35.8 \pm 0.2$ & $12.6 \pm 0.2$ \\
\hline NGC 7592 West & $23: 18: 21.84$ & $-04: 24: 57.1$ & 5.3 & $350.8 \pm 6.5$ & $1422.0 \pm 14.9$ & $297.4 \pm 2.3$ & $171.1 \pm 3.4$ & $53.3 \pm 0.4$ & $13.6 \pm 0.5$ \\
\hline NGC 7592 East & $23: 18: 22.64$ & $-04: 24: 58.1$ & 5.3 & $726.6 \pm 6.8$ & $2446.7 \pm 18.6$ & $508.8 \pm 4.6$ & $224.3 \pm 4.1$ & $67.2 \pm 0.6$ & $23.9 \pm 0.5$ \\
\hline NGC 7592 South & $23: 18: 22.16$ & $-04: 25: 08.6$ & 1.4 & $36.5 \pm 2.5$ & $132.3 \pm 6.2$ & $29.5 \pm 1.3$ & $23.5 \pm 4.0$ & $4.2 \pm 0.2$ & $3.4 \pm 0.9$ \\
\hline
\end{tabular}


Table 3

$\mathrm{H}_{2}$ Fluxes

\begin{tabular}{|c|c|c|c|c|c|c|c|c|}
\hline Name & $\begin{array}{c}\mathrm{H}_{2} \mathrm{~S}(0) \\
28.22 \mu \mathrm{m}\end{array}$ & $\begin{array}{c}\mathrm{H}_{2} \mathrm{~S}(1) \\
17.03 \mu \mathrm{m}\end{array}$ & $\begin{array}{c}\mathrm{H}_{2} \mathrm{~S}(2) \\
12.28 \mu \mathrm{m}\end{array}$ & $\begin{array}{l}\mathrm{H}_{2} \mathrm{~S}(3) \\
9.66 \mu \mathrm{m}\end{array}$ & $\begin{array}{l}\mathrm{H}_{2} \mathrm{~S}(4) \\
8.03 \mu \mathrm{m}\end{array}$ & $\begin{array}{l}\mathrm{H}_{2} \mathrm{~S}(5) \\
6.91 \mu \mathrm{m}\end{array}$ & $\begin{array}{l}\mathrm{H}_{2} \mathrm{~S}(6) \\
6.11 \mu \mathrm{m}\end{array}$ & $\begin{array}{l}\mathrm{H}_{2} \mathrm{~S}(7) \\
5.51 \mu \mathrm{m}\end{array}$ \\
\hline NGC 520 System & $5.4 \pm 0.3$ & $11.2 \pm 0.2$ & $3.4 \pm 0.2$ & $10.9 \pm 0.7$ & $4.9 \pm 0.5$ & & & $2.8 \pm 0.7$ \\
\hline NGC 520 North & $0.7 \pm 0.1$ & $1.9 \pm 0.3$ & $0.7 \pm 0.4$ & $0.7 \pm 0.4$ & $2.6 \pm 1.2$ & & & $1.8 \pm 1.7$ \\
\hline NGC 520 South & $96.5 \pm 4.1$ & $86.4 \pm 0.8$ & $36.8 \pm 0.6$ & $139.4 \pm 3.1$ & $35.3 \pm 2.0$ & $21.5 \pm 4.1$ & & $3.3 \pm 2.9$ \\
\hline NGC 2623 & $1.5 \pm 0.3$ & $5.5 \pm 0.2$ & $1.1 \pm 0.2$ & $6.5 \pm 0.8$ & $1.9 \pm 0.6$ & & & $3.2 \pm 0.9$ \\
\hline NGC 3921 & $0.7 \pm 0.1$ & $5.4 \pm 0.3$ & $1.6 \pm 0.1$ & $3.5 \pm 0.5$ & $0.7 \pm 0.2$ & $1.4 \pm 0.4$ & $0.5 \pm 0.4$ & $0.2 \pm 0.4$ \\
\hline NGC 4676 System & $0.5 \pm 0.1$ & $2.9 \pm 0.3$ & $1.1 \pm 0.1$ & $1.7 \pm 0.2$ & $0.8 \pm 0.3$ & $1.2 \pm 0.4$ & & $0.6 \pm 0.4$ \\
\hline NGC 4676 North & $0.3 \pm 0.2$ & $5.1 \pm 0.6$ & $2.5 \pm 0.2$ & $4.1 \pm 0.4$ & $2.6 \pm 0.5$ & $2.9 \pm 1.0$ & & \\
\hline NGC 4676 South & $1.6 \pm 0.2$ & $9.6 \pm 0.5$ & $3.6 \pm 0.3$ & $6.7 \pm 0.4$ & $1.6 \pm 0.8$ & $4.3 \pm 1.2$ & & $2.2 \pm 1.0$ \\
\hline NGC 6240 & $16.9 \pm 0.8$ & $87.7 \pm 0.3$ & $25.1 \pm 0.2$ & $103.3 \pm 0.6$ & $36.0 \pm 0.6$ & $63.8 \pm 0.8$ & $12.3 \pm 0.7$ & $28.4 \pm 0.8$ \\
\hline NGC 6621 System & $0.5 \pm 0.0$ & $2.0 \pm 0.1$ & $0.9 \pm 0.1$ & $1.2 \pm 0.1$ & & & & $0.9 \pm 0.4$ \\
\hline NGC 6621 North & $2.0 \pm 0.1$ & $5.8 \pm 0.3$ & $8.8 \pm 0.3$ & $7.6 \pm 0.4$ & $6.7 \pm 0.9$ & $17.2 \pm 1.4$ & & \\
\hline NGC 6621 South & $0.9 \pm 0.1$ & $3.5 \pm 0.3$ & $0.3 \pm 0.2$ & $2.7 \pm 0.2$ & $1.3 \pm 0.5$ & $0.6 \pm 0.2$ & & \\
\hline NGC $6621 \mathrm{Mid}$ & $0.7 \pm 0.2$ & $2.3 \pm 0.4$ & $0.6 \pm 0.2$ & $0.4 \pm 0.3$ & & & & $5.0 \pm 2.4$ \\
\hline NGC 7252 & $0.4 \pm 0.2$ & $4.8 \pm 0.3$ & $2.0 \pm 0.2$ & $3.7 \pm 0.4$ & $1.0 \pm 0.6$ & $0.9 \pm 0.8$ & & \\
\hline NGC 7592 System & $1.8 \pm 0.1$ & $7.4 \pm 0.2$ & $3.8 \pm 0.2$ & $7.4 \pm 0.5$ & $1.8 \pm 0.8$ & $2.6 \pm 1.1$ & & \\
\hline NGC 7592 West & $2.4 \pm 0.2$ & $14.1 \pm 0.4$ & $9.1 \pm 0.4$ & $13.0 \pm 1.0$ & $6.8 \pm 1.3$ & $9.6 \pm 2.0$ & & $1.3 \pm 1.6$ \\
\hline NGC 7592 East & $1.6 \pm 0.2$ & $7.3 \pm 0.4$ & $4.3 \pm 0.5$ & $11.5 \pm 1.1$ & & $6.5 \pm 2.7$ & & \\
\hline NGC 7592 South & $1.8 \pm 0.3$ & $2.1 \pm 0.7$ & $0.2 \pm 0.2$ & $0.2 \pm 0.4$ & $0.4 \pm 0.7$ & 0.7 & & \\
\hline
\end{tabular}

Notes. Overview of the integrated $\mathrm{H}_{2}$ line strengths of the $\mathrm{H}_{2}$ components for all merger components. The center and size of the regions over which they are integrated are the same as in Table 2. All units are in $10^{-9} \mathrm{~W} \mathrm{~m}^{-2} \mathrm{sr}^{-1}$.

Table 4

$\mathrm{H}_{2}$ Temperatures and Masses

\begin{tabular}{|c|c|c|c|c|}
\hline Name & $\begin{array}{c}T_{\text {warm }} \\
(\mathrm{K})\end{array}$ & $\begin{array}{l}T_{\text {hot }} \\
(\mathrm{K})\end{array}$ & $\begin{array}{c}\mathrm{H}_{2} \text { Mass Warm } \\
\left(10^{6} M_{\odot}\right)\end{array}$ & $\begin{array}{c}\mathrm{H}_{2} \text { Mass Hot } \\
\left(10^{6} M_{\odot}\right)\end{array}$ \\
\hline NGC 520 System & $221.4 \pm 10.5$ & $877.1 \pm 42.3$ & $16.0 \pm 0.2$ & $1.2 \pm 0.3$ \\
\hline NGC 520 North & $246.1 \pm 93.7$ & & $0.2 \pm 0.1$ & \\
\hline NGC 520 South & $87.7 \pm 0.2$ & $550.1 \pm 7.0$ & & $10.3 \pm 8.9$ \\
\hline NGC 2623 & $162.6 \pm 38.8$ & $969.7 \pm 67.8$ & $118.3 \pm 5.1$ & $2.6 \pm 0.7$ \\
\hline NGC 3921 & $241.7 \pm 17.4$ & $664.5 \pm 82.5$ & $33.4 \pm 1.9$ & $5.1 \pm 10.0$ \\
\hline NGC 4676 System & $286.5 \pm 18.7$ & $1041.8 \pm 188.0$ & $21.0 \pm 1.9$ & $1.0 \pm 0.6$ \\
\hline NGC 4676 North & $309.4 \pm 27.5$ & $1091.8 \pm 366.2$ & $4.9 \pm 0.6$ & \\
\hline NGC 4676 South & $291.7 \pm 17.0$ & $990.2 \pm 136.7$ & $9.2 \pm 0.5$ & $0.7 \pm 0.3$ \\
\hline NGC 6240 & $184.9 \pm 1.9$ & $746.1 \pm 3.2$ & $1608.1 \pm 6.2$ & $279.5 \pm 8.0$ \\
\hline NGC 6621 System & $316.8 \pm 16.8$ & & $43.8 \pm 1.8$ & \\
\hline NGC 6621 North & $380.8 \pm 6.0$ & & $8.6 \pm 0.4$ & \\
\hline NGC $6621 \mathrm{Mid}$ & $252.8 \pm 27.6$ & & $2.9 \pm 0.5$ & \\
\hline NGC 7252 & $296.1 \pm 37.0$ & $663.7 \pm 240.8$ & $6.5 \pm 0.4$ & \\
\hline NGC 7592 System & $333.8 \pm 26.2$ & $847.3 \pm 318.4$ & $14.9 \pm 0.5$ & \\
\hline NGC 7592 West & $348.1 \pm 14.0$ & $1078.9 \pm 213.3$ & $5.0 \pm 0.1$ & $0.1 \pm 0.2$ \\
\hline NGC 7592 East & $339.4 \pm 66.3$ & $838.2 \pm 305.8$ & $2.7 \pm 0.2$ & \\
\hline
\end{tabular}

Note. Temperatures and masses of the $\mathrm{H}_{2}$ emission derived by fitting the excitation diagram with a two-component model.

[Ne III], the measured [Ne III]/[Ne II] line flux ratio is sensitive to the average effective temperature of the ionizing stars, and hence might provide a measure of the hardness of the radiation field.

The spectral maps of the $[\mathrm{Ne}$ III]/[Ne II] line ratios for our sample are shown in Figure 8. For all our galaxies the nuclei have the smallest [Ne III]/[Ne II] ratios (see also right panel of Figure 7), while the circumnuclear regions tend to show (a factor of 2-4) larger ratios. Intuitively, this is in contrast to the expectation of a decrease of the hardening of the radiation field with distance to the center due to the small number of high energy photons available at large distances to ionize $\mathrm{Ne}^{+}$ to $\mathrm{Ne}^{2+}$. However, our result is in agreement with other recent observations: Pereira-Santaella et al. (2010) have found that the minimum of the $[\mathrm{Ne}$ III $] /[\mathrm{Ne}$ II] ratio tends to be located at the nuclei for a sample of 15 LIRGs observed with Spitzer IRS; Thornley et al. (2000) have shown that H II galaxies have a nuclear $[\mathrm{Ne}$ III] $/[\mathrm{Ne}$ II] ratio of a factor $\sim 2-3$ smaller than those measured in the extra-nuclear regions. A similar behavior of increasing $[\mathrm{Ne} \mathrm{III}] /[\mathrm{Ne} \mathrm{II}]$ ratios with increasing galactocentric distances has been observed in the nearby galaxies NGC 253 (Devost et al. 2004), M82 (Beirão et al. 2008), and NGC 891 (Rand et al. 2008). Galactic H II regions follow the same trend, with $\mathrm{H}$ II regions at increasing distances from the galactic nucleus showing larger $[\mathrm{Ne} \mathrm{III}] /[\mathrm{Ne} \mathrm{II}]$ ratios (Giveon et al. 2002). Our results confirm this trend, even if dust extinction corrections are taken into account as a function of position in the galaxies (using the SCREEN model in PAHFIT). Metallicityrelated effects are unlikely to be the dominant cause of the relative increase of [Ne III] strength outside the central regions 

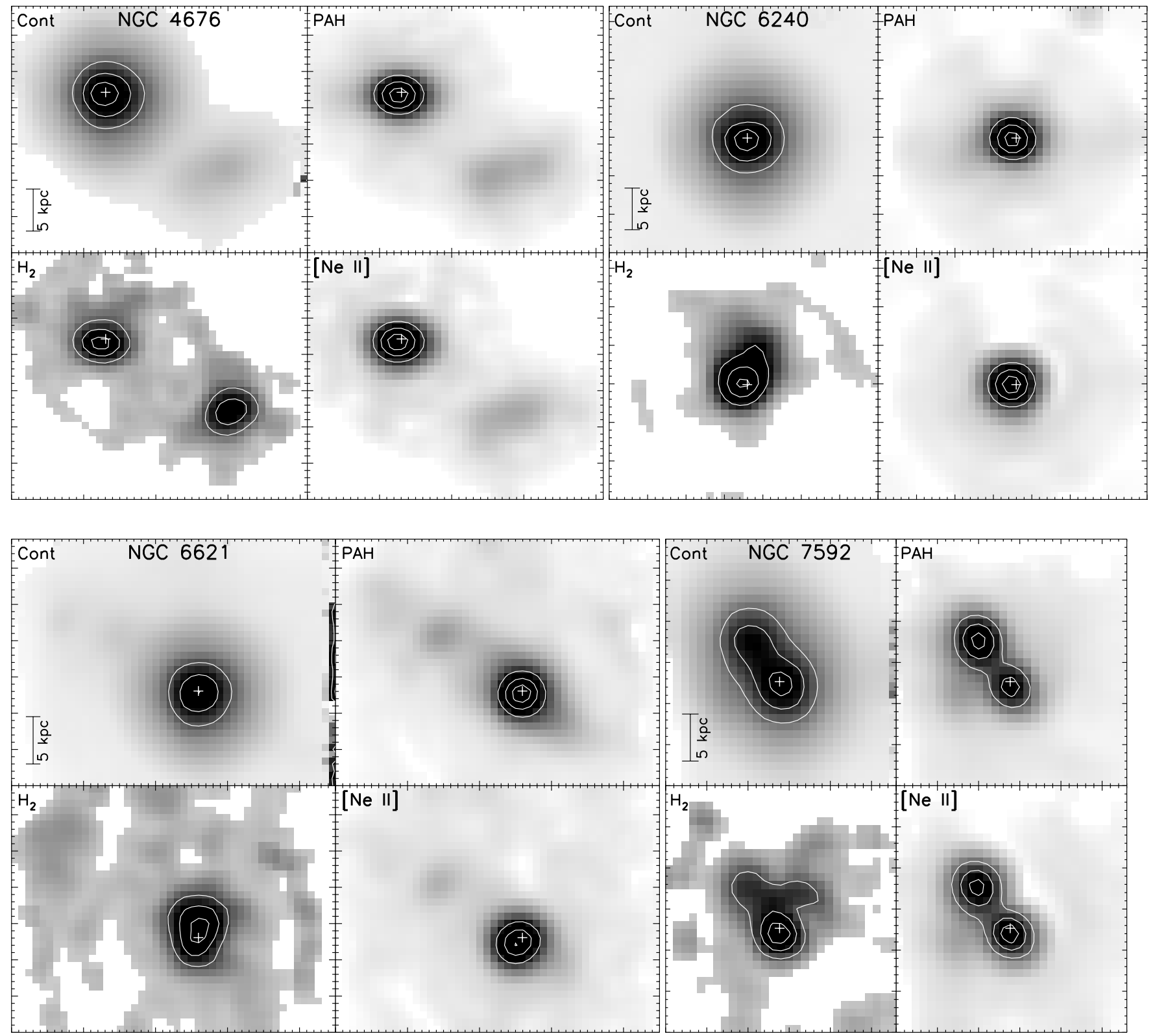

Figure 4. Main mid-IR feature maps with contours, from left to right and top to bottom: the integrated mid-IR continuum (5.3-15 $\mu \mathrm{m})$ as measured with PAHFIT, the combined PAH features, the combined $\mathrm{H}_{2}$ emission features, and the [Ne II] emission (see the text). The center is marked with a plus symbol signifying the peak in the integrated mid-IR continuum and the tickmarks correspond to 1 pixel intervals (1".85). The north and east directions in each frame of this figure are the same as in the corresponding frame of Figure 2.

of these galaxies (Snijders et al. 2007; Pereira-Santaella et al. 2010). A more likely explanation may be that a fraction of the most massive stars are hidden in ultracompact $\mathrm{H}$ II regions (Rigby \& Rieke 2004) due to high dust densities in the nuclear regions, leading, e.g., to a decrease of the $[\mathrm{Ne} \mathrm{III}] /[\mathrm{Ne}$ II $]$ ratio by a factor of $\sim 2$ if the density increases from $10^{3}$ to $10^{5} \mathrm{~cm}^{-3}$ (Snijders et al. 2007). Another explanation for this behavior might be attributed to the variation in the age of the stellar populations, with a larger contribution of the old stellar population in the nuclei (e.g., due to stellar bulges), subsequently decreasing the average stellar age (and ionization) toward the center.

\subsubsection{Comparison of Global Mid-IR Properties to Normal Star Formation Galaxies}

The SINGS galaxies, being mostly isolated, star-forming systems with a large range in IR power, are a perfect sample against which to compare the mid-IR properties of our major mergers. In particular one might expect to find different PAH populations between these two samples due to, e.g., a different interplay between radiation field and the ISM. Since the PAH $11.3 \mu \mathrm{m}$ feature is thought to be produced by neutral PAHs, whereas the $7.7 \mu \mathrm{m}$ feature arises primarily from PAH ions (Allamandola et al. 1999; Draine \& Li 2001), a change in the neutral fraction should change the observed flux ratio. Harder radiation fields are also more efficient at destroying small PAH grains, an effect that may dominate the center of our galaxies. For all of our objects, the ranges of the integrated (i.e., global) PAH strength ratios $6.2 / 7.7 \mu \mathrm{m}$ and $11.3 / 17 \mu \mathrm{m}$ are in the range of [0.2-0.4] and [1-2.5], respectively. We find a range in the ratio $7.7 / 11.3 \mathrm{PAH}$ bands of [1-6] and a range in [Ne III]/[Ne II] of [0.1-1]. The respective ranges and distributions of the ratios PAH 6.2/7.7 $\mu \mathrm{m}, 11.3 / 17 \mu \mathrm{m}$, and [Ne III]/[Ne II] are very similar to interband strength ratios found in the nuclei of 22 


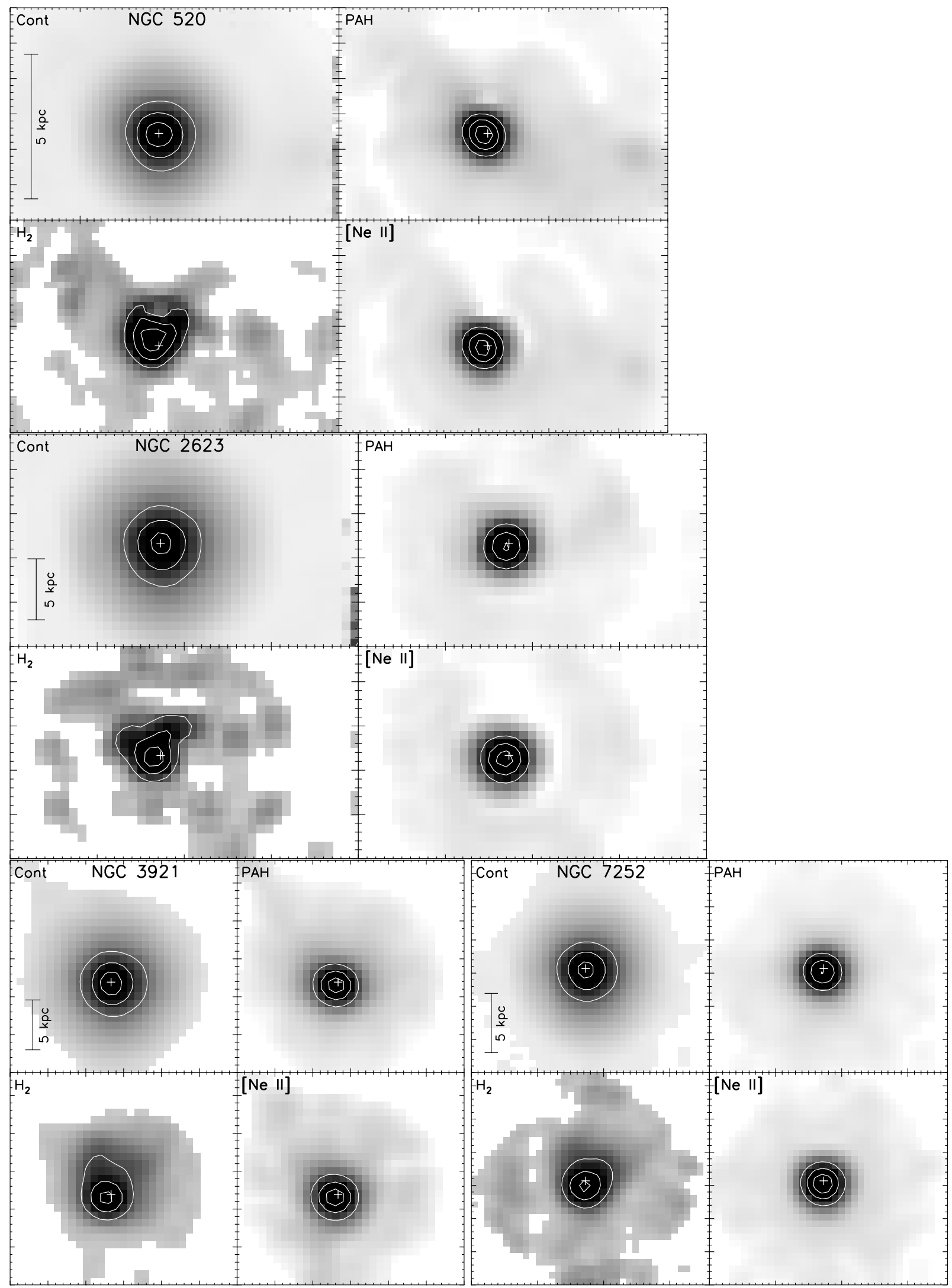

Figure 4. (Continued)

starburst galaxies (Brandl et al. 2006) and for a large sample of SINGS galaxies (Smith et al. 2007), including normal star formation galaxies and low-luminosity AGNs (Seyfert and
LINER). This suggests that the physical properties in major mergers are, at least on global scales, similar to those in normal starburst galaxies, but can be very different on local scales 

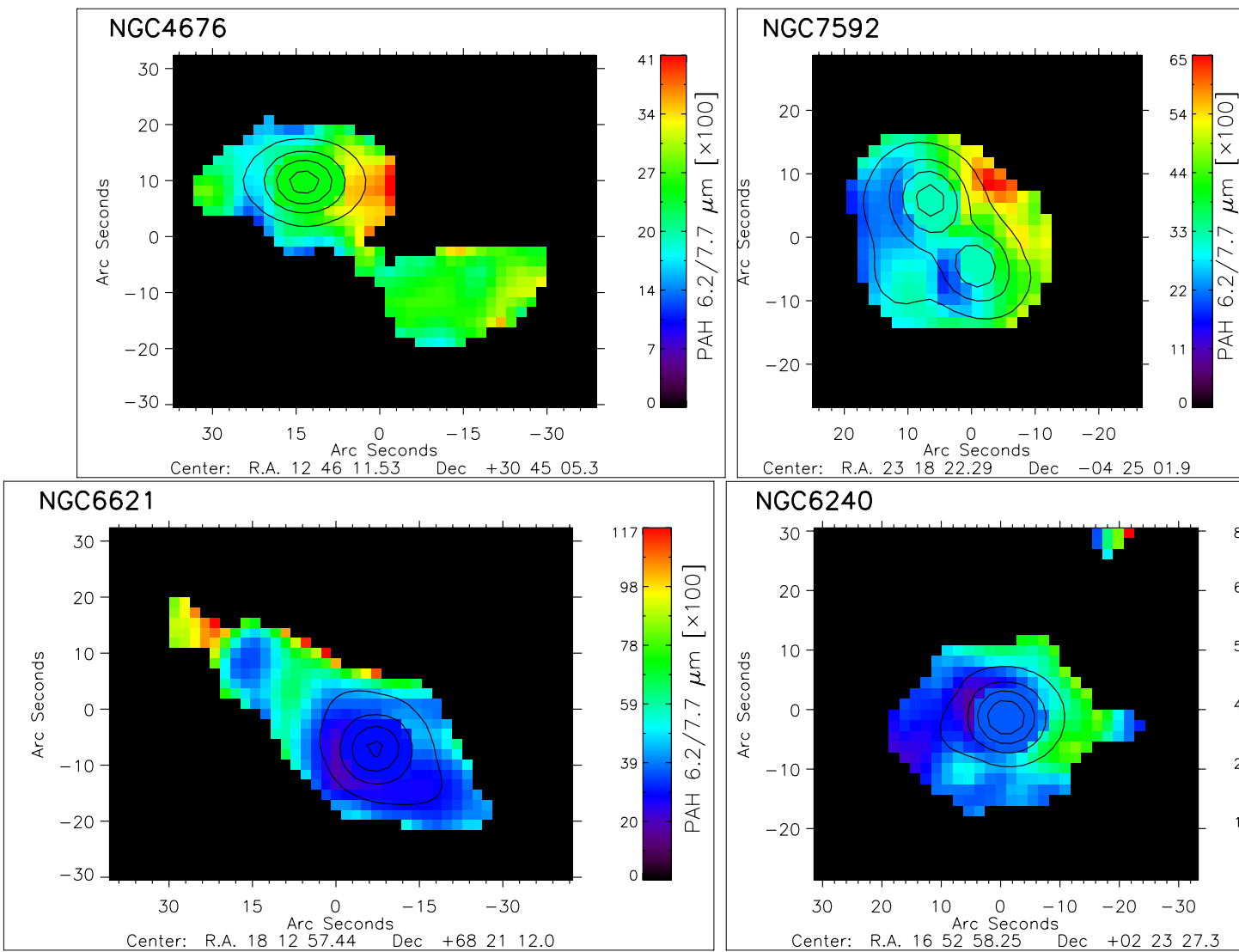

Center: R.A. 23
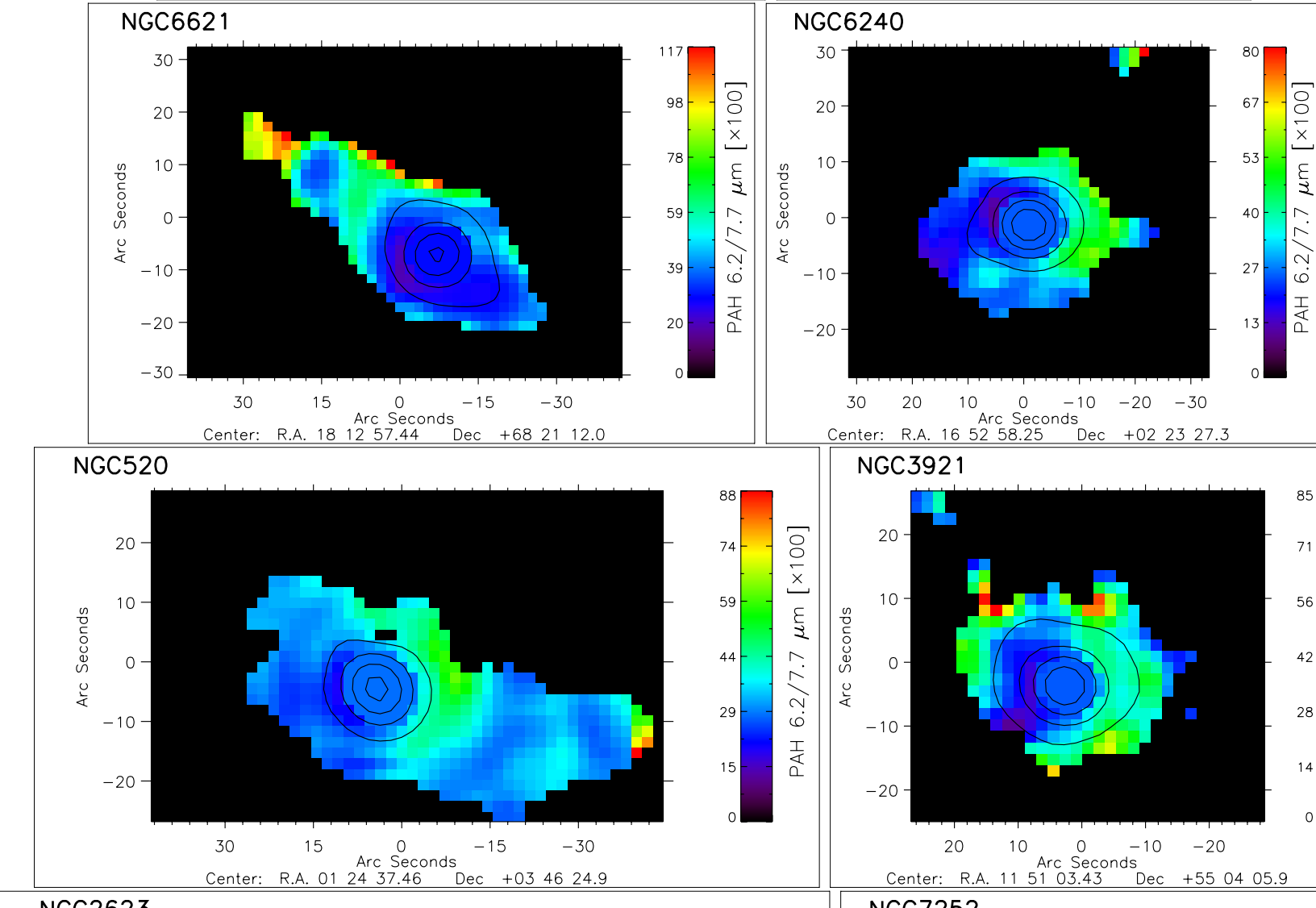

Center: R.A. 165258.25 Dec +02 2327.3
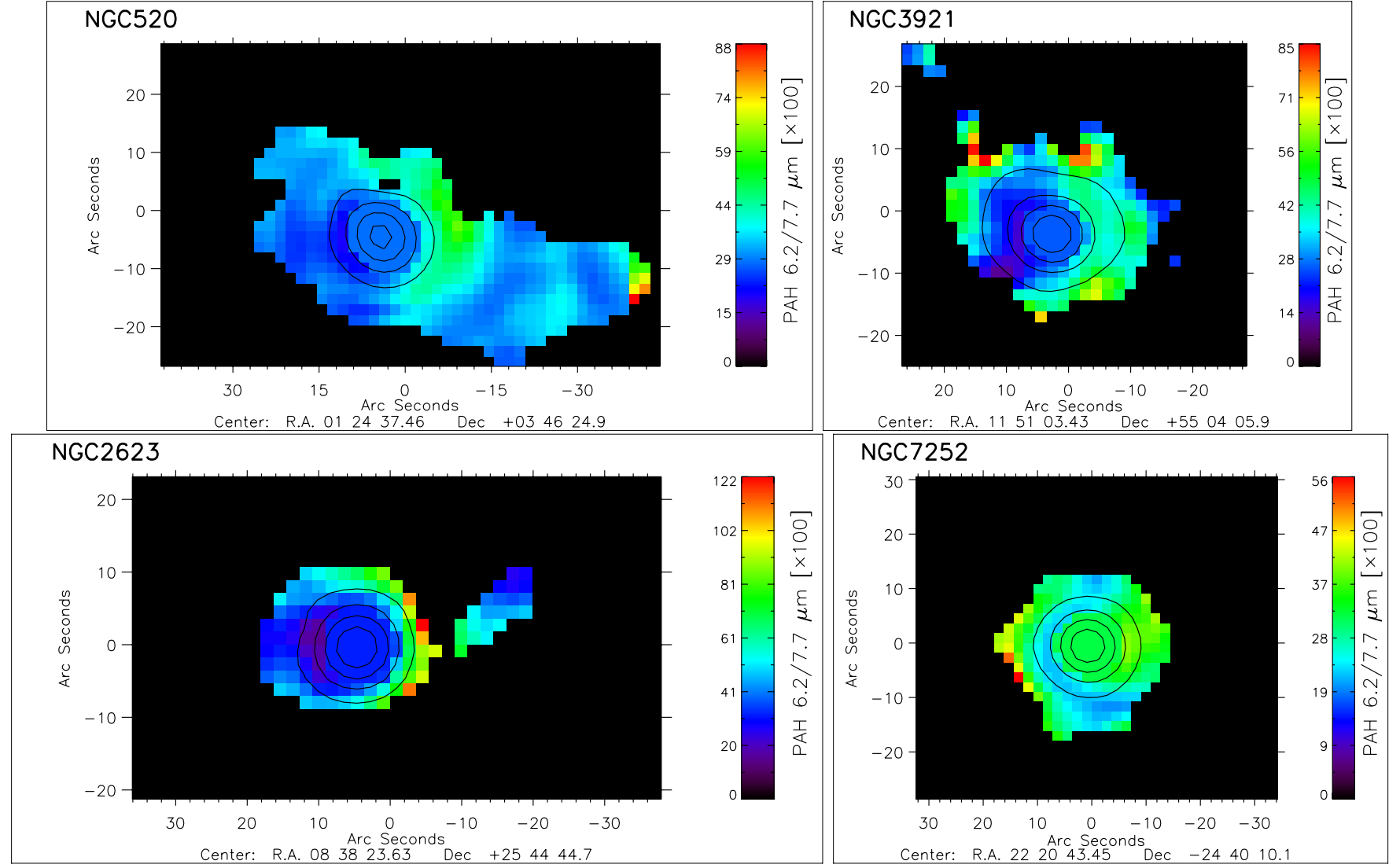

Figure 5. Ratio map of PAH 6.2/7.7 $\mu \mathrm{m}$ overlaid with the combined PAH emission $(6.2,7.7$, and $11.3 \mu \mathrm{m})$ in contours. The north and east directions in each frame of this figure are the same as in the corresponding frame of Figure 2.

(A color version of this figure is available in the online journal.) 

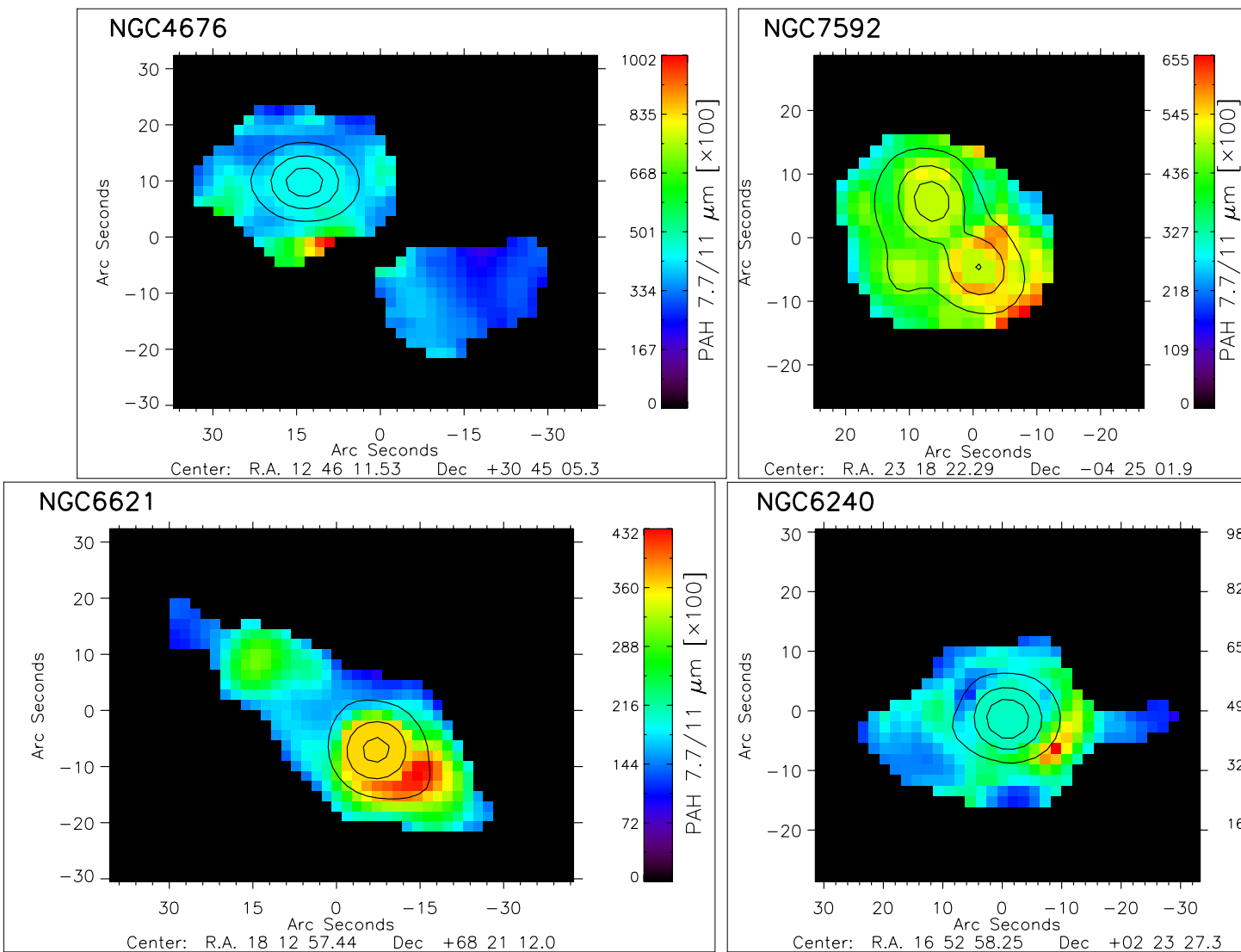

\section{NGC6240}
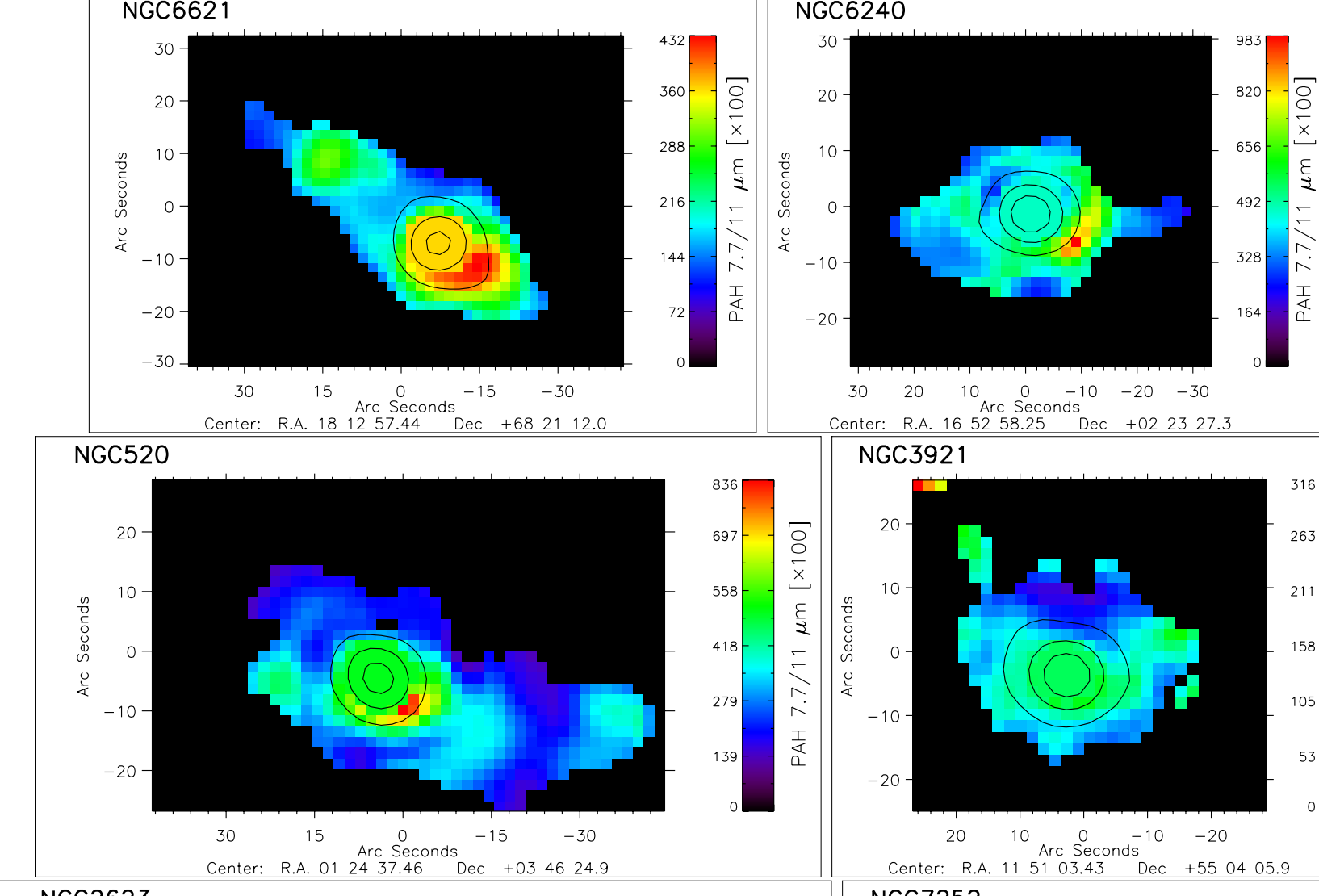

Center: R.A. 165258.25 Dec +022327.3
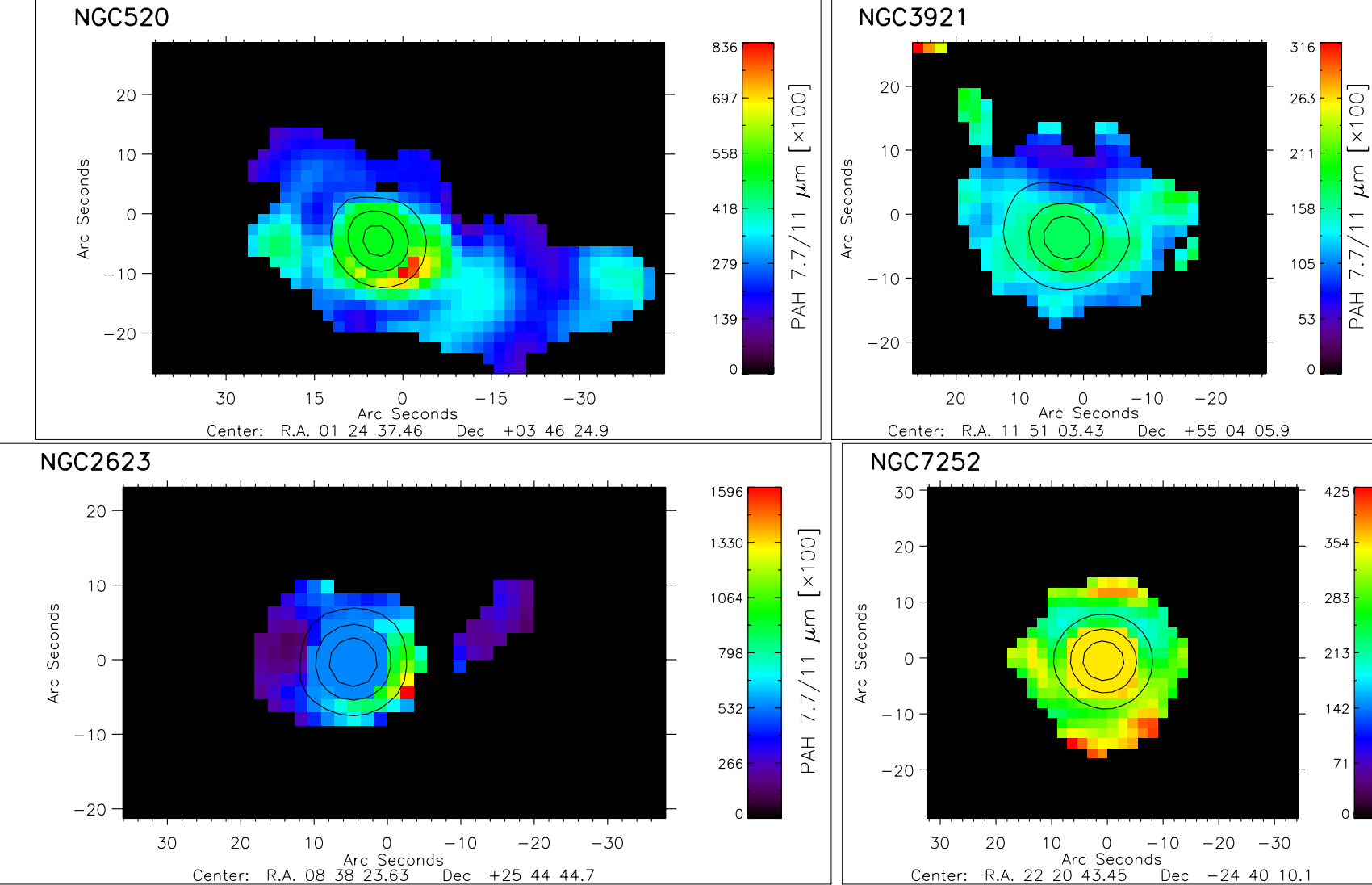

Center: R.A. 115103.43 Dec +550405.9

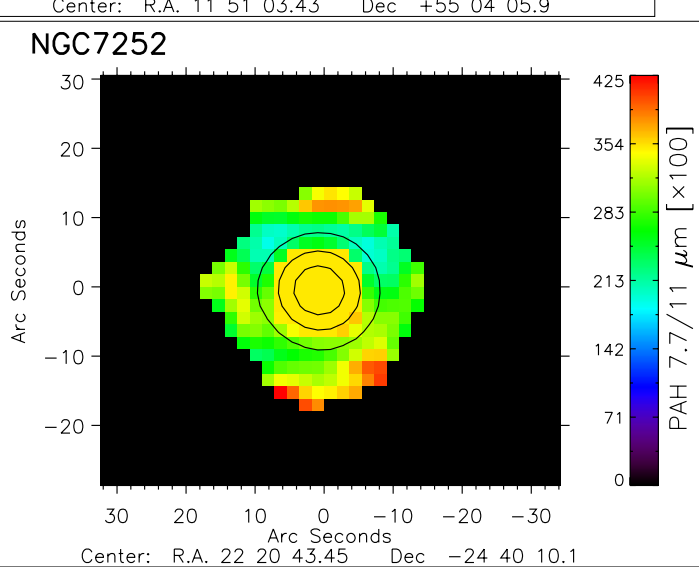

Figure 6. Ratio map of PAH 7.7/11.3 $\mu \mathrm{m}$ overlaid with the combined PAH emission $(6.2,7.7$, and $11.3 \mu \mathrm{m})$ in contours. The north and east directions in each frame of this figure are the same as in the corresponding frame of Figure 2.

(A color version of this figure is available in the online journal.) 

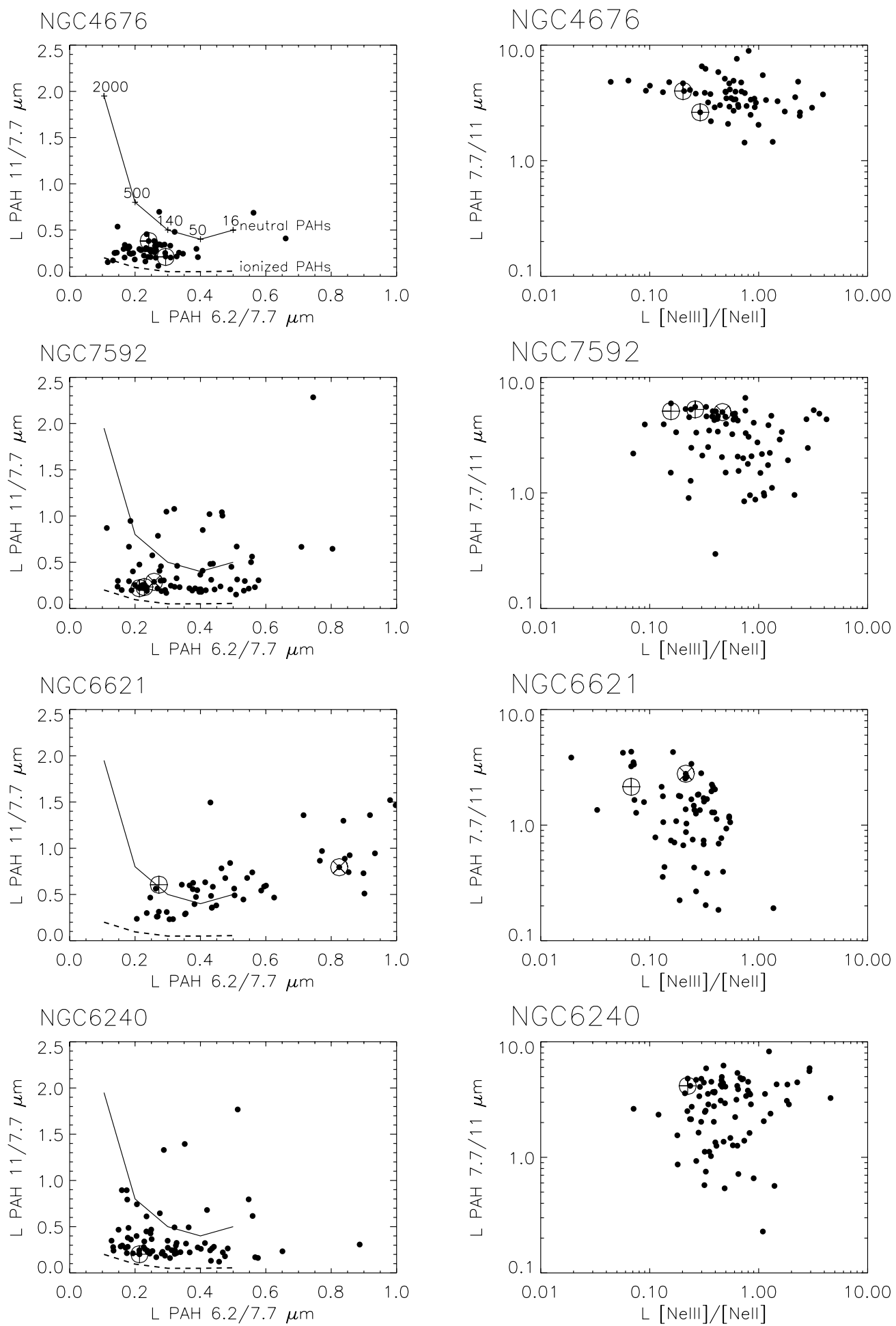

Figure 7. Left: variation of interband strength ratios of the PAH features ( $x$-axis: PAH $6.2 / 7.7 \mu \mathrm{m}, y$-axis:PAH $11.3 / 7.7 \mu \mathrm{m})$ over $3 \times 3$ pixels $(5$ ". $6 \times 5$ "' 6 , corresponding to the aperture) within the merger system (see Figures 5 and 6). The model by Draine \& Li (2001) for neutral and ionized PAHs (from a few tens to thousands of carbon atoms as marked in the top left panel) is overlaid as solid and dashed line, respectively. The nuclei of the galaxies (central aperture) are indicated as a circle with plus marker. Right: variation of interband strength ratios of the PAH features at $7.7 \mu \mathrm{m}$ relative to the $11.3 \mu \mathrm{m}$ band with the line ratio [Ne III]/[Ne II].

within the individual merger systems as shown in the following sections. However, we do not see any global ratios of [Ne III]/ [Ne II] $>2$ in our sample, which is the case for five lower metallicity galaxies with H II-dominated nuclei in the SINGS sample (Smith et al. 2007) as well as in most blue compact dwarfs and in galaxies with a strong AGN component (Hao et al. 2009).

\subsection{Properties and Distribution of the Warm Molecular Gas Component}

\subsubsection{The Distribution of the Warm Molecular Gas}

Excitation of the warm molecular gas observed in the rotational emission lines of $\mathrm{H}_{2}(\mathrm{~S}(0)-\mathrm{S}(7))$ can commonly arise from at least three different mechanisms: UV excitation 

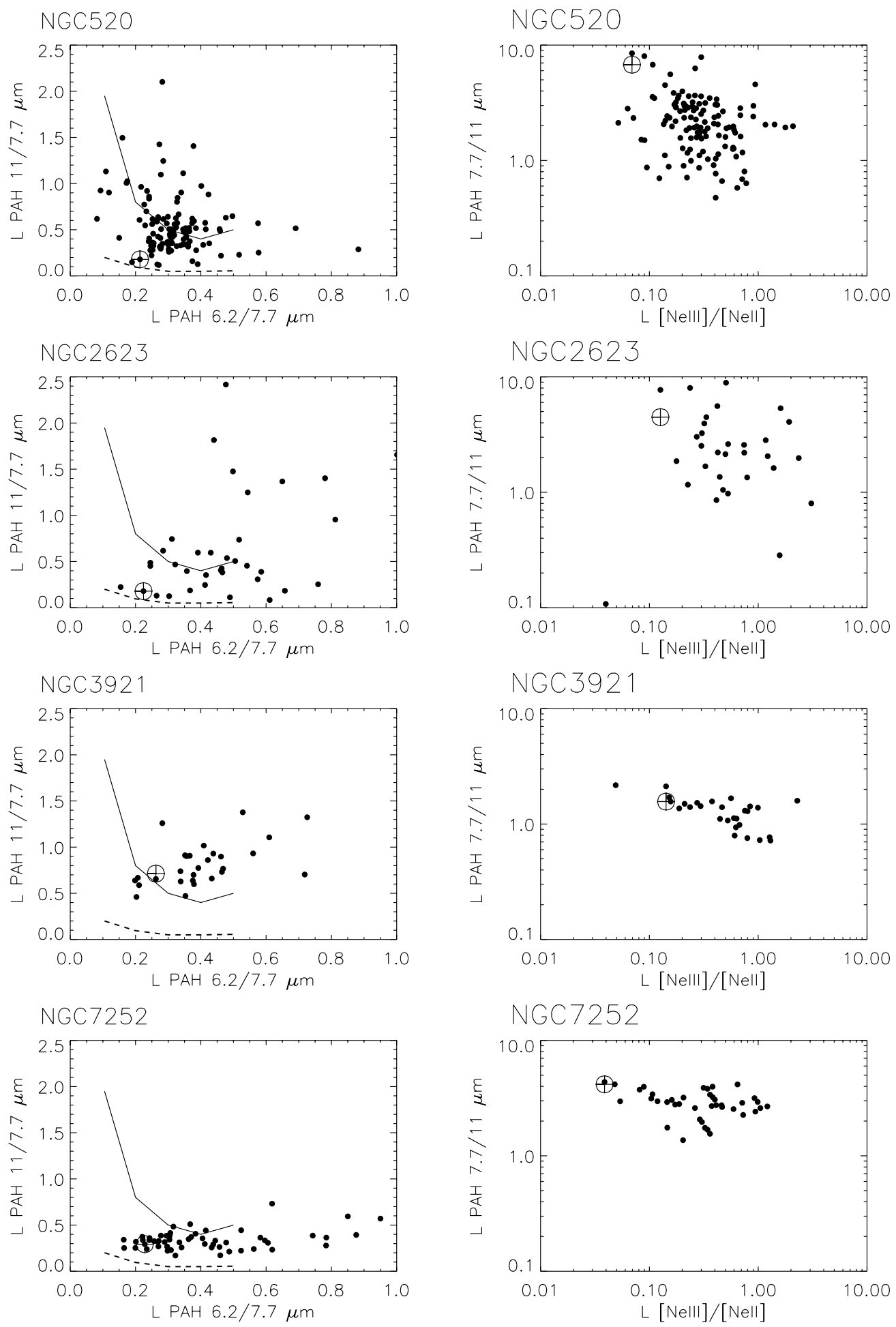

Figure 7. (Continued)

in PDRs surrounding or adjacent to the H II regions; shocks that collisionally excite the $\mathrm{H}_{2}$ molecules; or hard X-ray photons capable of penetrating the molecular clouds and heating large columns of gas (Lepp \& McCray 1983; Maloney et al. 1996). Observationally separating these primary excitation mechanisms is not trivial in other galaxies: high UV radiation as well as high-velocity gas motions are present in both the nuclei of active galaxies and, to a lesser extent, in starbursts. UV radiation can originate either from the existing stellar population, from current star-forming processes, or the AGN accretion disk. On the other hand, shock excitation can originate from the central engine, an outflow due to the large number of supernova remnants, or finally from gas motions and cloud collisions due to galaxy-galaxy interactions (Shull \& Hollenbach 1978; Draine et al. 1983). In addition, the low rotational transitions arise from collisionally excited warm gas, which complicates the distinction even further. However, if $\mathrm{H}_{2}$ emission is excited mainly by UV radiation in PDRs, the fluxes of $\mathrm{H}_{2}$ lines and PAH features should correlate closely, since PAH emission features arise from the same mechanism in PDRs. 

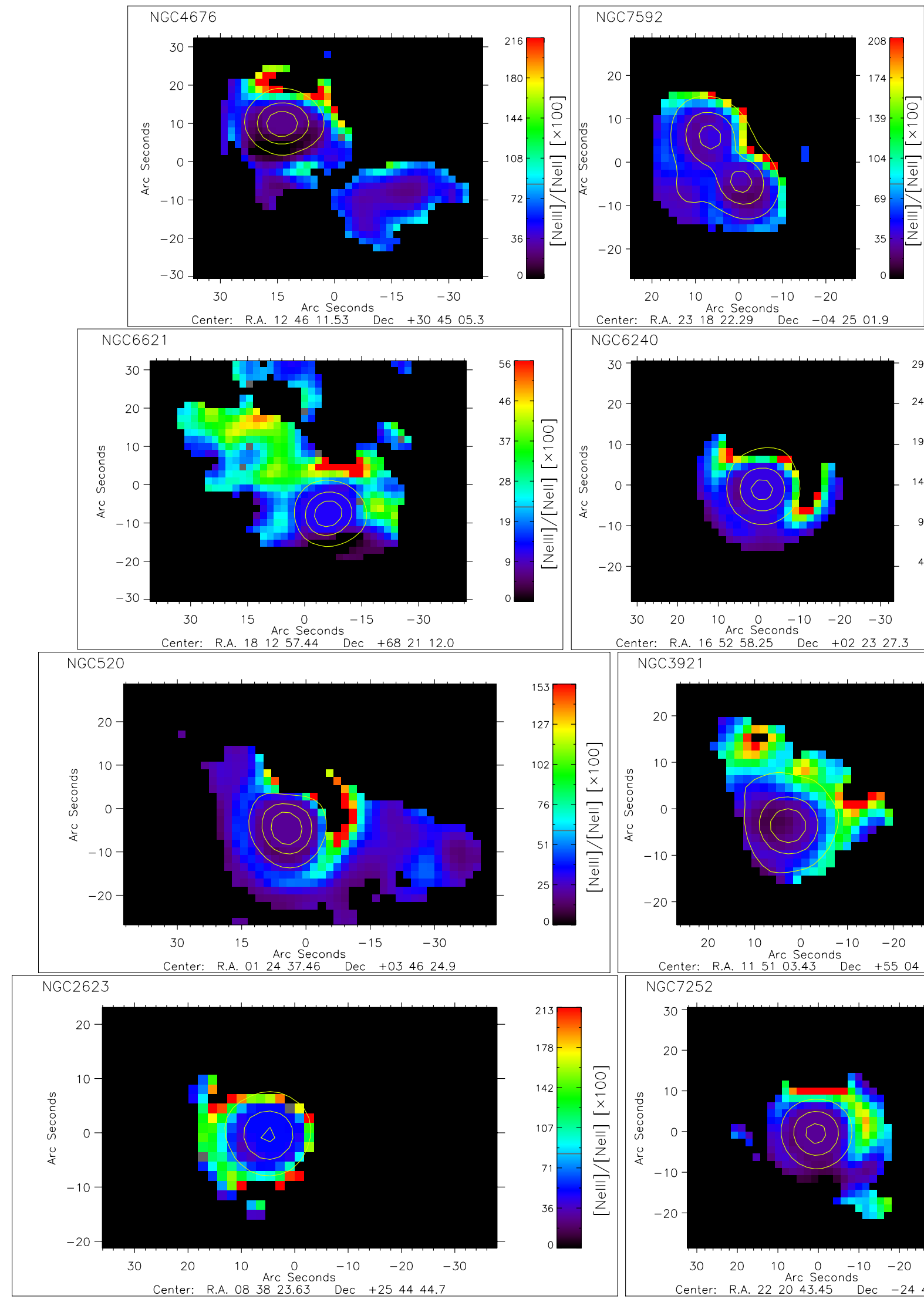

Center: R.A.

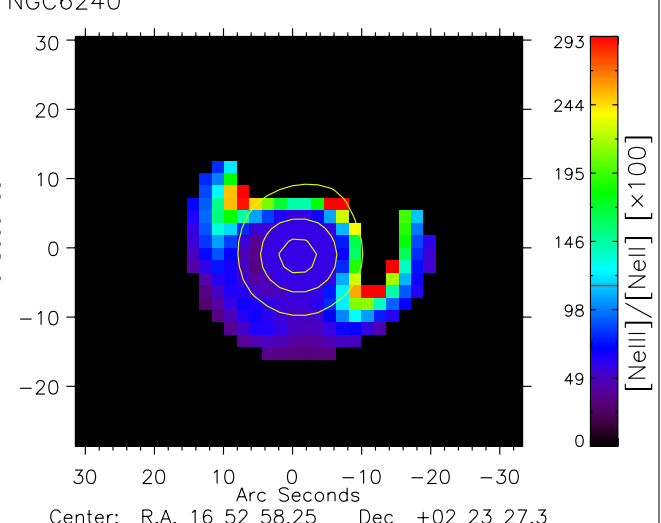

Center: R.A. 165258.25 Dec +02 2327.3 NGC3921

Figure 8. [Ne III]/[Ne II] line flux ratio maps for the Toomre sequence galaxies. The contours indicate the sum of the [Ne III] and [Ne II] flux. The north and east directions in each frame of this figure are the same as in the corresponding frame of Figure 2.

(A color version of this figure is available in the online journal.) 


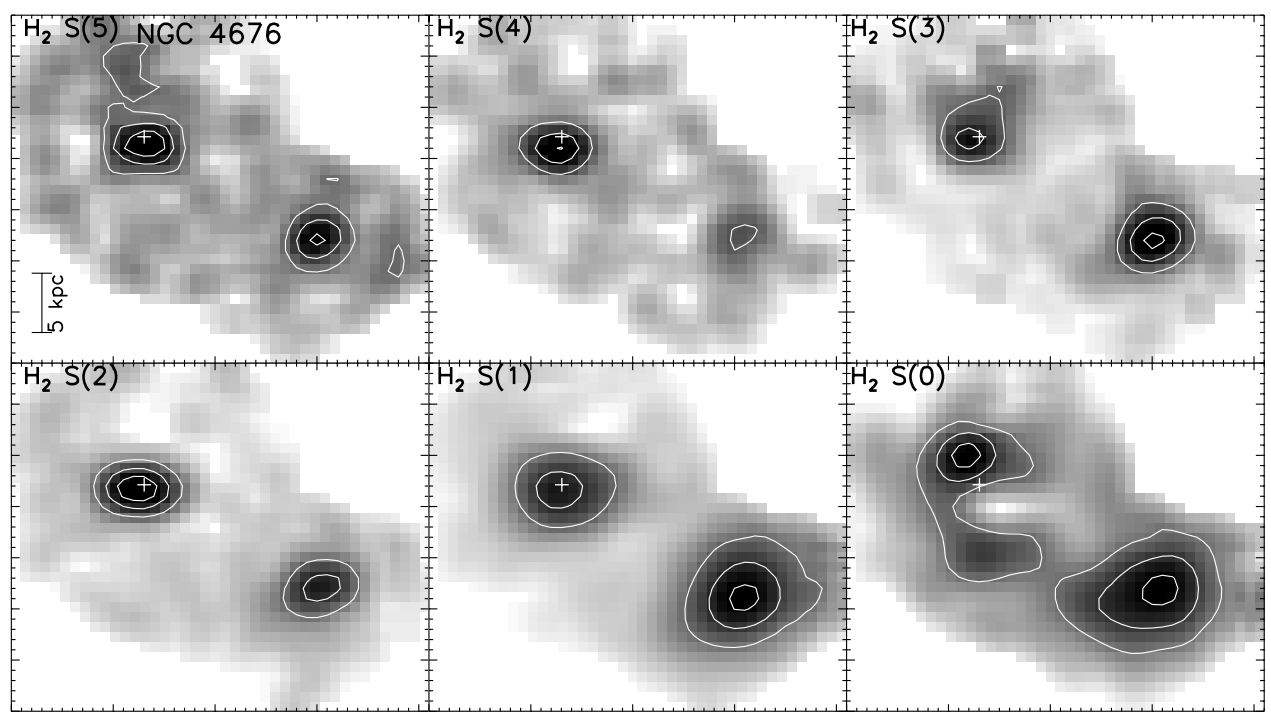

Figure 9. $\mathrm{H}_{2}$ feature maps with contours (from $\mathrm{S}(5)$ at top left to $\mathrm{S}(0)$ at bottom right). The plus marker indicates the central peak in the mid-IR continuum emission. The north and east directions in each frame of this figure are the same as in the corresponding frame of Figure 2.

(The complete figure set ( 8 images) is available in the online journal.)

In Figure 9, we have mapped the emission from several $\mathrm{H}_{2}$ rotational lines for each merger system. Our comparison between the flux distribution of the strongest $\mathrm{H}_{2}$ emission line (the extinction corrected $9.7 \mu \mathrm{m} \mathrm{H}_{2}$ emission line) and $\mathrm{PAH}$ (using the combined main PAH features in the SL wavelength range at $6.1,7.7$, and $11.3 \mu \mathrm{m}$ ) shows that $(1) \mathrm{H}_{2}$ has typically a larger extent than $\mathrm{PAH}$ with an average ratio of $\mathrm{H}_{2} / \mathrm{PAH}$ radius of 1.5 (ranging from 1.1 to 1.9, including PSF size corrections), (2) some galaxies show a significantly different $\mathrm{H}_{2} / \mathrm{PAH}$ flux ratio between different merger components, most significantly in the early merger systems NGC 4676 and NGC 7592 (see Section 3.5), and (3) at least one of our galaxies (NGC 3921; see also for optical spectroscopy, Schweizer et al. 1996) shows signs of a tail of $\mathrm{H}_{2}$ emission not associated with a strong PAH feature (see Figure 4).

Five galaxies (NGC 7592, NGC 6621, NGC 6240, NGC 520, NGC 2623) show in Figure 9 an offset of the peak $\mathrm{H}_{2}$ emission at $9.66 \mu \mathrm{m}$ from the stellar nucleus, which is not seen in any $\mathrm{PAH}$ or other $\mathrm{H}_{2}$ features (e.g., $\mathrm{S}(2)$ and $\mathrm{S}(4)$ ). One possible explanation for this offset is an underestimation of dust extinction (silicate absorption at $9.7 \mu \mathrm{m}$ ), which is strongest at the center of the galaxy, and hence suppresses the apparent $\mathrm{H}_{2}$ flux at $9.66 \mu \mathrm{m}$. However, even without the $9.7 \mu \mathrm{m} \mathrm{H}_{2}$ emission line, we find spatial deviations from a constant relation between the warm molecular gas $\left(\mathrm{H}_{2}\right)$ and PAH (as described above), indicating that, at least in some merger galaxies, different physical conditions are present in the ISM (e.g., electron density, gas temperature, or UV radiation field intensity; for a review see Galliano et al. 2008). The nature of the variation in the $\mathrm{H}_{2} / \mathrm{PAH}$ ratio will be discussed in more detail in Section 3.3.4.

\subsubsection{Spatially Integrated Temperatures and Masses}

Temperatures and masses of the warm molecular hydrogen $(T<2000 \mathrm{~K})$ are derived from the pure rotational $\mathrm{H}_{2}$ lines. Figure 10 shows the excitation diagrams using the extinction corrected line fluxes (see Table 3) for different regions of the merger systems. In all cases an ortho-to-para ratio of 3 is assumed (Rigopoulou et al. 2002; Roussel et al. 2007). The excitation diagram is basically a plot of the natural logarithm of the column density $N_{i}$ divided by the statistical weight $g(i)$

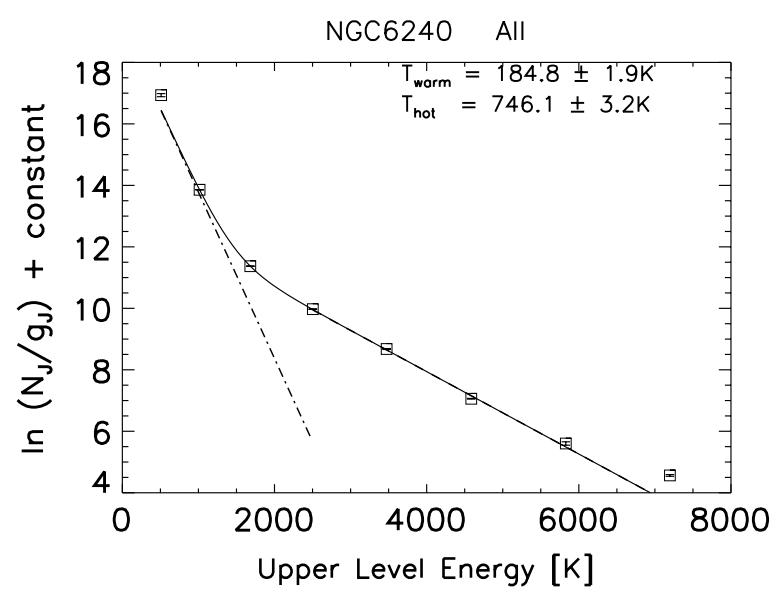

Figure 10. $\mathrm{H}_{2}$ excitation diagram for NGC 6240, showing the upper level population divided by the level degeneracy as a function of upper level energy (for the lines observed with the IRS). The lines are measured with PAHFIT, including a correction for extinction. Uncertainties are indicated as vertical bars for each line. A double exponential profile fit (solid line) takes into account two temperature components (see the text), separately indicated as dashed and dashed dotted lines.

(The complete figure set (17 images) is available in the online journal.)

in the upper level of each transition against the energy level $E_{i}$. A detailed description can be found in Roussel et al. (2007), including a discussion about the implications of using different ortho-to-para ratios.

The excitation temperature of the line-emitting gas is the reciprocal of the slope of the excitation diagram. If the $\mathrm{H}_{2}$ gas is characterized by a single temperature (and assuming a single ortho-to-para ratio), the data will lie on a straight line, corresponding to a single component fit. We find that a simple single temperature fit does not work for most of our galaxies, since our data show a change of the slope with energy level. This is due to the fact that the gas phase is in reality more complex and consists of various components at different temperatures. The $S(4)$ to $S(7)$ lines (upper energy level from $\sim 3500$ to $7200 \mathrm{~K}$ ) show in general a much smaller slope than the slope inferred from the $S(0)$ and $S(1)$ lines (upper energy 


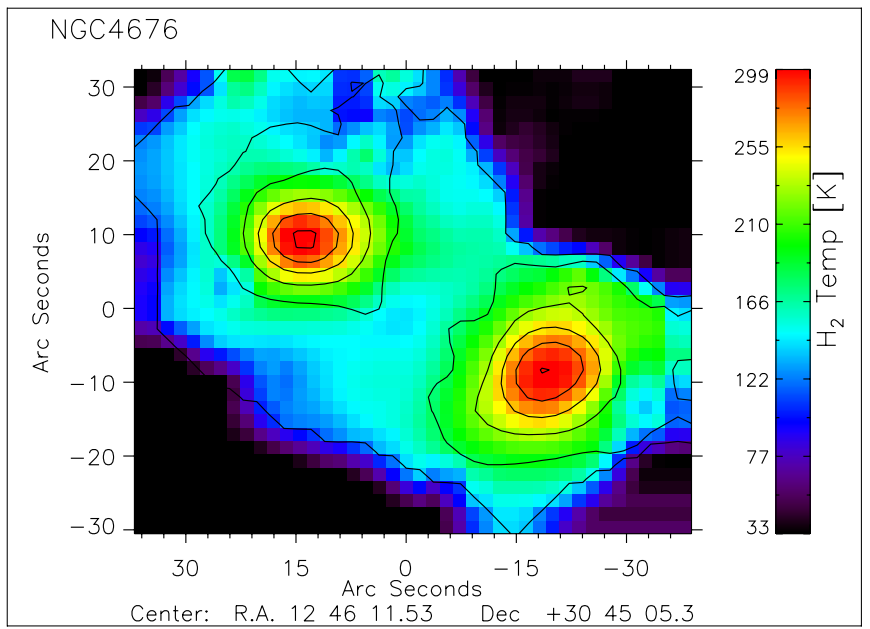

Figure 11. Single-component $\mathrm{H}_{2}$ temperature map (in colors) with the combined $\mathrm{H}_{2}$ emission (in contours) for NGC 4676.

(A color version and the complete figure set ( 8 images) of this figure are available in the online journal.)

level of 500 to $1000 \mathrm{~K}$ ), representing a "hot" and "warm" temperature component of the gas, respectively. To take into account these two temperature components, we fitted a double exponential profile to our data, given as

$$
\frac{N_{i}}{g_{i}}=c_{0}+c_{1} \exp \left(E_{i} / T_{\text {warm }}\right)+c_{2} \exp \left(E_{i} / T_{\text {hot }}\right),
$$

where $N_{i}$ is the number of molecules, $g_{i}$ is the statistical weight of each state, $E_{i}$ is the upper energy level, $c_{0-2}$ are constants, and $T_{\text {warm }}$ and $T_{\text {hot }}$ are the two excitation temperatures. This fit has the advantage that it simultaneously fits two components, rather than starting with two independent temperature estimates using different lines and subsequently subtracting the contribution of the hot component from the warm component.

Figure 10 shows the excitation diagrams and the fits to the warm and hot $\mathrm{H}_{2}$ phases for the different regions of our merger systems. For all our objects, except for NGC 6621, the excitation diagram of the entire merger system could be well approximated by a two temperature component fit. Only the warm component could be measured for NGC 6621 because the $\mathrm{H}_{2}$ lines $\mathrm{S}(4)-\mathrm{S}(6)$ are not detected. We find for our sample a mean temperature of the warm $\mathrm{H}_{2}$ phase of $255 \mathrm{~K}$, but with a large variance between different regions, ranging from $87 \mathrm{~K}$ (NGC 520 South) to $348 \mathrm{~K}$ (NGC 7592 South). The mean temperature of the hot phase for our sample is $830 \mathrm{~K}$, ranging from $550 \mathrm{~K}$ (NGC 520 South) to 1079 K (NGC 7592 South). In comparison to other studies, our average excitation temperature of the warm component seems to be slightly larger than in the nuclear region of a sample of SINGS galaxies ( $200 \mathrm{~K}$; Roussel et al. 2007), but smaller than in the nuclear region of starburst galaxies $(\sim 360 \mathrm{~K}$; Bernard-Salas et al. 2009) and ULIRGs ( 336 K; Higdon et al. 2006). Note that this comparison does neither take into account the different sizes over which the excitation temperature is averaged nor the large differences in warm molecular masses involved.

We used the two excitation temperatures $T_{\text {warm }}$ and $T_{\text {hot }}$ to estimate the total warm and hot gas masses (Rigopoulou et al. 2002), $M_{\text {warm }}$ and $M_{\text {hot }}$, in our sources, which are presented in Table 4. The total mass of the warm component (median $\left.M_{\text {warm }}=3.3 \times 10^{7} M_{\odot}\right)$ is in general at least an order of magnitude larger than the total mass of the hot component (median $M_{\text {hot }}=0.1 \times 10^{7} M_{\odot}$ ).

\subsubsection{Temperature Maps}

To reveal spatial variations of the $\mathrm{H}_{2}$ temperature in the merger components, we compute $\mathrm{H}_{2}$ temperature maps as shown in Figure 11. In only one galaxy, NGC 6240, does the $\mathrm{H}_{2}$ line emission from the hot component $(\mathrm{S}(4)-\mathrm{S}(7))$ have a sufficient $\mathrm{S} / \mathrm{N}$ per pixel to employ a two component temperature fit per pixel. (Figure 12). For all other galaxies a one-component fit (per pixel) reveals similar results on the warm component as the two-component fit (per pixel) due to a limited number of significant $\mathrm{H}_{2}$ lines detected per pixel. Note that this does not imply that these galaxies have only one temperature component per pixel, since our spatially integrated flux measurements (with higher $\mathrm{S} / \mathrm{N}$ ) show that almost all galaxies have at least two temperature components (Section 3.3.2). Hence, the absolute temperature scale is not necessarily equal to the spatially integrated temperature, which is due to smaller $\mathrm{S} / \mathrm{Ns}$ on pixel scale. The absolute temperatures are given in the previous section (see also Table 4) and we focus instead on the temperature distribution in this section. In general, we find an increase in temperature toward the peak of the combined $\mathrm{H}_{2}$ flux, which is in most cases aligned with the center of the main galaxy component. We find no $\mathrm{H}_{2}$ hot spots between merger galaxies. An exception is NGC 6240 where we find a faint second temperature peak $(500 \mathrm{~K}), \sim 7 \mathrm{kpc}$ eastward,
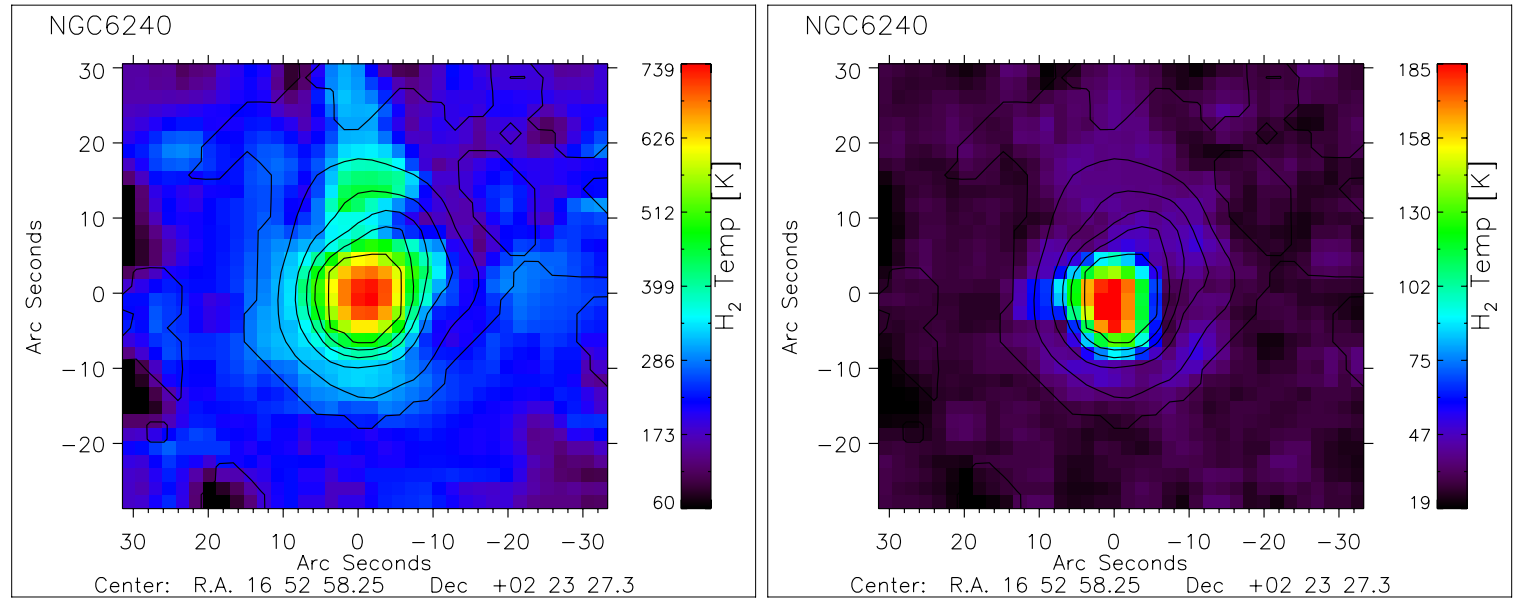

Figure 12. Two $\mathrm{H}_{2}$ temperature maps (left: hot, right: warm component) for NGC 6240, with the combined $\mathrm{H}_{2}$ emission in contours.

(A color version of this figure is available in the online journal.) 
not in alignment with the $\mathrm{H}_{2}$ flux intensity nor any other mid-IR feature or NIR concentration. Although both gas temperature components are mapped, only the "hot" component exhibits this secondary temperature peak.

\subsubsection{Excitation Mechanisms: Evidence for an Intrinsic Relation between $\mathrm{H}_{2}$ and $\mathrm{PAH}$ Emission}

One of the major excitation sources in starburst galaxies is the far-ultraviolet (FUV) radiation of massive stars in PDRs, with photon energies between 6 and $13.6 \mathrm{eV}$ (Hollenbach \& Tielens 1997). PDRs are attenuated by the absorption and scattering of UV photons from a hot star, generating several layers with different temperatures and material components. In general, $\mathrm{PAH}$ and $\mathrm{H}_{2}$ can be expected to be co-spatial since both species can be excited by FUV photons (as discussed in Section 3.3.1). Galactic studies of the molecular hydrogen emission in PDRs suggest that the formation of $\mathrm{H}_{2}$ occurs on the surfaces of PAHs (see, e.g., Habart et al. 2003; Velusamy \& Langer 2008). A similar correlation between $\mathrm{H}_{2}$ and $\mathrm{PAH}$ emission is seen in external galaxies (Rigopoulou et al. 2002; Roussel et al. 2007). In particular, Roussel et al. (2007) found for normal star-forming galaxies, on average, a constant flux ratio of $\mathrm{H}_{2}$ (S0-S2)/PAH $\sim 0.008$ over a large range of total $\mathrm{H}_{2}$ fluxes, suggesting relatively constant physical conditions in PDRs. However, this study was limited (1) to the average emission of only the H II nuclei and complexes within the kiloparsec-scale circumnuclear region for each galaxy and (2) by the fact that the power emitted in the aromatic bands was estimated from the IRAC4 filter rather than via direct spectroscopic measurement of PAH emission lines. On the other hand, a recent study of ULIRGs suggests that the $\mathrm{H}_{2}$ emission does not come from PAH regions (PDR's) and originates outside the dust obscured regions due to shocks in the surrounding material (Zakamska 2010).

Since $\mathrm{H}_{2}$ emission can arise from at least three different mechanisms (UV-excitation in PDRs, shocks, or hard X-ray radiation from an AGN), one important question is whether there is one single excitation mechanism that dominates the radiation power emitted in the mid-IR or a mix of various excitation mechanisms in mergers. In principle, studying the relation between $\mathrm{PAH}$ and rotational $\mathrm{H}_{2}$ emission over various spatial scales allows us to constrain the role of PDRs, as both PAH and $\mathrm{H}_{2}$ are tightly correlated with the physical conditions in PDRs (in particular with the radiation field intensity $G_{0}$ and the hydrogen density $n_{\mathrm{H}}$ ). Since most of the warm $\mathrm{H}_{2}$, at the lowest rotational temperatures, emits primarily in the $S(0)$ to $\mathrm{S}$ (2) lines, whereas the $\mathrm{S}(3)$ line emission has a noticeably higher contribution from hotter $\mathrm{H}_{2}$, probably indicating more mixed excitation, a useful measure of the warm $\mathrm{H}_{2}$ in starforming galaxies is the sum of the $S(0)$ to $S(2)$ lines (Roussel et al. 2007).

Here, we study the relation between the combined $\mathrm{H}_{2}$ flux (from S0 to S2) and the sum of the four main PAH bands (at 6.2, 7.7., 11.3, and $17 \mu \mathrm{m}$ ) for various spatial locations in major merger systems (integrated over apertures of $3 \times 3$ pixels or $5^{\prime \prime} .6 \times 5.6$, corresponding to $\sim 2 \mathrm{kpc}$ at a mean distance of $79 \mathrm{Mpc}$ ). In Figure 13, we present the total flux ratios $\left.\mathrm{H}_{2}(\mathrm{~S} 0-\mathrm{S} 2)\right] / \mathrm{PAH}$ as a function of the total $\mathrm{H}_{2}(\mathrm{~S} 0-\mathrm{S} 2)$ luminosity, while Figure 14 shows the logarithmic relation between $\mathrm{H}_{2}(\mathrm{~S} 0-\mathrm{S} 2)$ and $\mathrm{PAH}$ for each galaxy. The following results are derived and compared to the center of normal star-forming galaxies (Roussel et al. 2007).

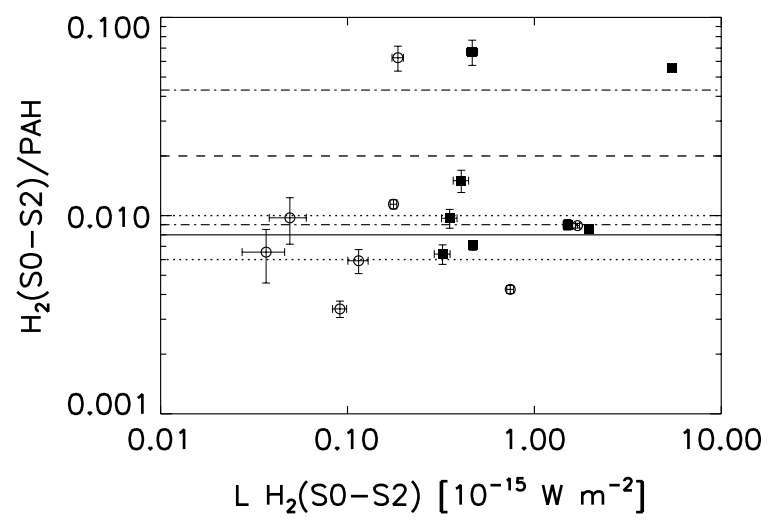

Figure 13. Ratio of the power emitted in the sum of the $\mathrm{H}_{2} \mathrm{~S}(0)$ to $\mathrm{S}(2)$ transitions to the power emitted in the main PAH bands (at 6.2, 7.7, 11.3, and $17 \mu \mathrm{m}$ ) over a large range of $\mathrm{H}_{2}$ luminosities. Measurements of the entire merger system are marked with filled squares while the individual merger components are marked with open circles. The solid (dotted) and dashed (dashed-dotted) line represents the mean (uncertainty) $\mathrm{H}_{2}(\mathrm{~S} 0-\mathrm{S} 2) / \mathrm{PAH}$ value measured for the center of normal star-forming galaxies and LINER/Seyfert nuclei in the SINGS sample, respectively (Roussel et al. 2007).

1. On global scales (integrated over the entire merger component), the majority of galaxies in our merger sample have similar total $\mathrm{H}_{2}(\mathrm{~S} 0-\mathrm{S} 2) / \mathrm{PAH}$ ratios (mean: $\left.0.008 \pm 0.004\right)$ as normal star-forming galaxies $\left(\mathrm{H}_{2}(\mathrm{~S} 0-\mathrm{S} 2) / \mathrm{PAH} \sim\right.$ 0.008). However, three galaxies in our sample (NGC 4676 South, NGC 6240, and NGC 3921) have a significant (a factor of 10) larger global ratio $\left(\mathrm{H}_{2}(\mathrm{SO}-\mathrm{S} 2) / \mathrm{PAH}=\right.$ $0.08)$, clearly separated from the rest of our sample and normal star-forming galaxies, and even larger than most AGN galaxies in the SINGS sample (mean $\mathrm{H}_{2}(\mathrm{~S} 0-\mathrm{S} 2) / \mathrm{PAH}=$ 0.016 for Seyfert and LINER host galaxies; Roussel et al. 2007).

2. On local scales (measuring different apertures within each merger component), we find an intrinsic relation between $\mathrm{H}_{2}$ and $\mathrm{PAH}$ flux as given by a power law,

$$
\log \left[\mathrm{H}_{2}(\mathrm{~S} 0-\mathrm{S} 2)\right]=k \log [\mathrm{PAH}]+\log [\text { const }] .
$$

All galaxies in our sample follow this power law with a similar slope component $k=0.61 \pm 0.05$ but a different constant (up to a factor of 10 difference).

3. Although the center of galaxies has the strongest radiation field and hosts an AGN for at least two galaxies in our sample (see Table 1), it follows the same power law as regions in the outer disk. We find no clear correlation between the presence of an $\mathrm{AGN}$ and the central $\mathrm{H}_{2} / \mathrm{PAH}$ ratio.

The presence of a power law suggests that $\mathrm{H}_{2}(\mathrm{~S} 0-\mathrm{S} 2)$ and $\mathrm{PAH}$ emission are tightly correlated, given as $\mathrm{H}_{2}(\mathrm{~S} 0-\mathrm{S} 2) \simeq$ $\mathrm{PAH}^{k}$, where $k$ is the scaling exponent and $a$ a constants. The scale invariance of the power law becomes clearer in the logarithmic form, $\log \left[\mathrm{H}_{2}(\mathrm{~S} 0-\mathrm{S} 2)\right]=k \log [\mathrm{PAH}]+\log [a]$, with a mean slope (exponent) $k=0.61( \pm 0.05)$ for our sample and a mean constant of $\log [a]=-4.0 \pm 0.6$. Note that the scatter around this slope typically increases toward the outer disk (smaller $\mathrm{S} / \mathrm{N}$ ) and that we cannot rule out a possible dependence of the power law on the resolved spatial scales. In a number of galaxies (NGC 6240, NGC 520, NGC 2623, and NGC 3921) the faint regions typically fall below our best-fit power law for the entire galaxy. These regions may have a steeper slope, closer to a constant value, implying the bright regions may have systematically less $\mathrm{H}_{2}$ emission for a given $\mathrm{PAH}$ flux than would 

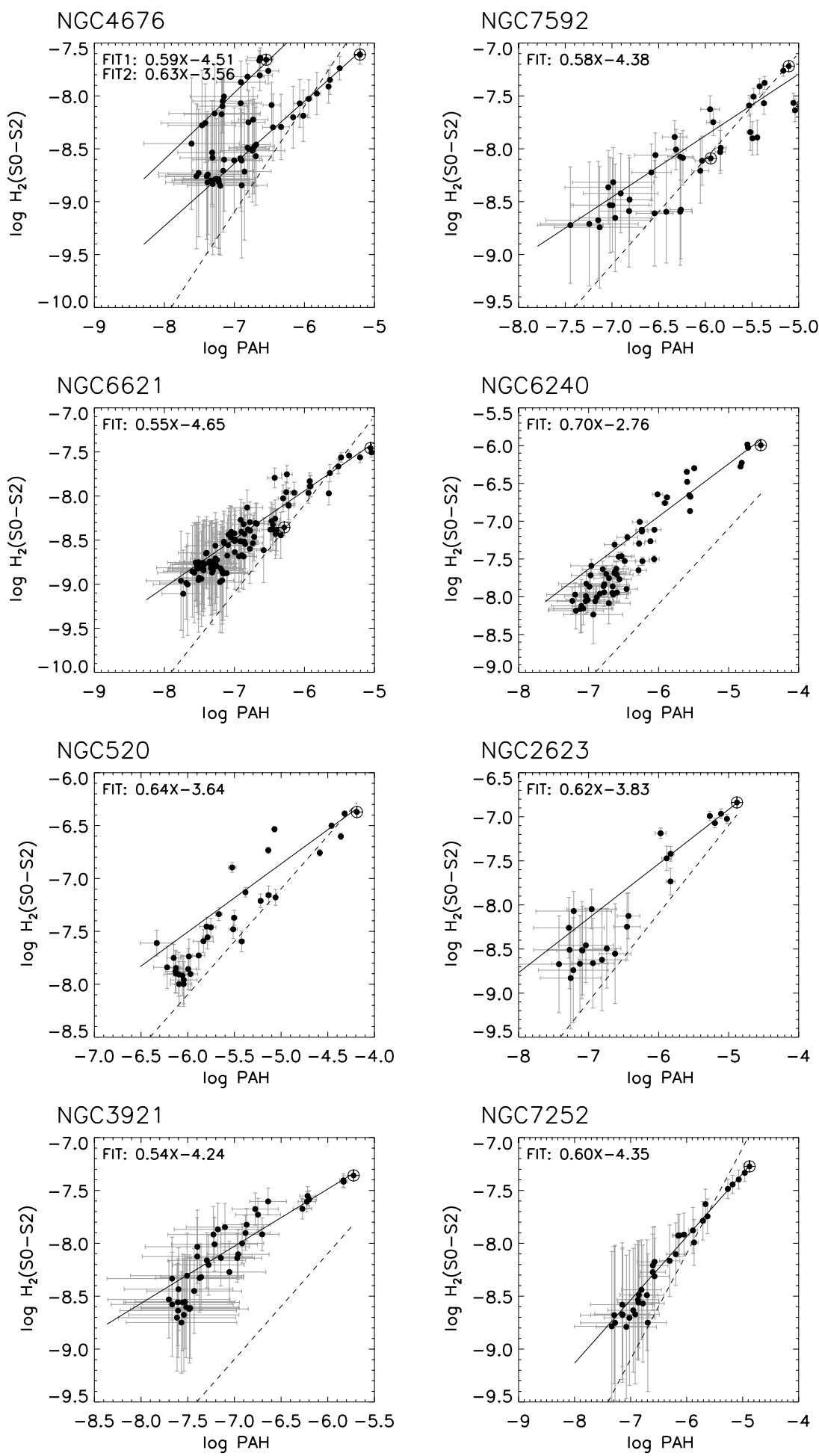

Figure 14. Power emitted in the sum of the $\mathrm{H}_{2} \mathrm{~S}(0)$ to $\mathrm{S}(2)$ transitions as a function of the power emitted in the main PAH bands (at $6.2,7.7,11.3$, and $17 \mu \mathrm{m}$ ) in logarithmic scales and in units of $\mathrm{W} \mathrm{m}^{-2} \mathrm{sr}^{-1}$. The crossed circles represent the central aperture of a merger component. The distribution is fitted using a power law (solid line), given as $\log \left[\mathrm{H}_{2}(\mathrm{~S} 0-\mathrm{S} 2]=k \times \log [\mathrm{PAH}]+c\right.$ with the slope $k$ and the constant $c$. The dashed line represents the constant ratio model for the center of normal star-forming galaxies, given as $\mathrm{H}_{2}(\mathrm{~S} 0-\mathrm{S} 2) / \mathrm{PAH}=0.008$ (Roussel et al. 2007).

be extrapolated from a fit to the faint regions in the outer disk. A higher extinction for the $\mathrm{H}_{2}$ lines might explain this, but higher $\mathrm{S} / \mathrm{N}$ data are required to confirm this change in slope.

To ensure that the slopes in Figure 14 and the fact that the nuclei display lower $\mathrm{H}_{2} / \mathrm{PAH}$ ratios than the galactic disks was not the result of having higher spatial resolution in the dominant
PAH feature $(7.7 \mu \mathrm{m})$ compared with the dominant $\mathrm{H}_{2}$ feature $(17 \mu \mathrm{m})$ because of the diffraction-limited nature of Spitzer, we have smoothed the SL PAH maps by a factor of three to match $\mathrm{SL}$ to $\mathrm{LL}$, and re-calculated the $\mathrm{H}_{2} / \mathrm{PAH}$ ratios for all galaxies. After this process, the slopes in Figure 14 and the ratios in the nuclei show a change of less than $3 \%$, suggesting that the effects 


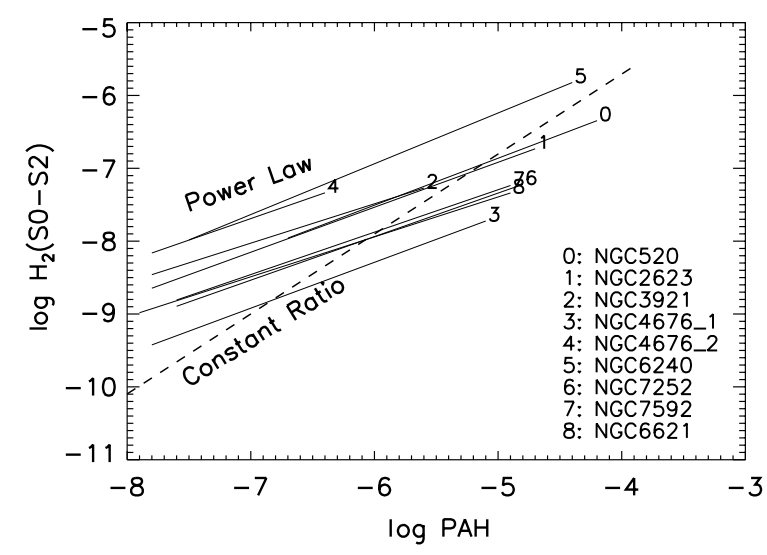

Figure 15. Overview of the power laws $\left(\log \left[\mathrm{H}_{2}(\mathrm{~S} 0-\mathrm{S} 2]=k \times \log [\mathrm{PAH}]+c\right)\right.$ as derived in Figure 14 for each galaxy (solid lines). The dashed line represents the constant ratio model for the center of normal star-forming galaxies, given as $\mathrm{H}_{2}(\mathrm{~S} 0-\mathrm{S} 2) / \mathrm{PAH}=0.008$ (Roussel et al. 2007), clearly demonstrating that a constant ratio (i.e., $k=1$ ) cannot describe the measured relation between $\mathrm{PAH}$ and $\mathrm{H}_{2}$ (mean $\left.k=0.61 \pm 0.05\right)$.

we are seeing, namely, slopes significantly less than unity and lower $\mathrm{H}_{2} / \mathrm{PAH}$ ratios in the nuclei than in the disk, are real. Moreover, we find a similar trend for most of our galaxies if we calculate the $\mathrm{H}_{2} / \mathrm{PAH}$ ratios using the $\mathrm{H}_{2} \mathrm{~S}(3)$ line $(9.7 \mu \mathrm{m})$ instead of $\mathrm{H}_{2}(\mathrm{~S} 0-\mathrm{S} 2)$, but with a typically much larger scatter and uncertainty (likely due to the fact that the $\mathrm{S}(3)$ emission line is more affected by uncertainties in the dust extinction correction and that it has a noticeably higher contribution from hotter $\mathrm{H}_{2}$ with a more mixed excitation as mentioned above).

While the study of Roussel et al. (2007) suggests a linear relation with a constant ratio $\mathrm{H}_{2}(\mathrm{~S} 0-\mathrm{S} 2) / \mathrm{PAH}=$ 0.008 for the center of normal star-forming galaxies, we find that a constant ratio (i.e., $k=1$ ) cannot describe the relation between $\mathrm{PAH}$ and $\mathrm{H}_{2}$ emission over the entire galaxies' disk as demonstrated in Figure 15. The nuclei of the galaxies tend to be closest to the Roussel et al. (2007) value while the regions in the disk tend to have higher $\mathrm{H}_{2} / \mathrm{PAH}$ ratios (increased $\mathrm{H}_{2} / \mathrm{PAH}$ ratios at radii of 5 to $15 \mathrm{kpc}$ have been also found in the edge-on disk galaxies NGC 4565 and NGC 5907; Laine et al. 2010). Instead, the intrinsic relation between $\mathrm{PAH}$ and $\mathrm{H}_{2}$ emission follows a power law from the center to the outskirts of major mergers. This tight correlation and the fact that we find a similar slope (exponent) for diverse merger systems suggest a common underlying excitation mechanism. The most likely mechanism is FUV excitation of $\mathrm{PAH}$ and $\mathrm{H}_{2}$ gas in PDRs, since other mechanisms, such as shocks due to supernovae or AGNs, would (1) not show the same behavior over all spatial scales and (2) are not expected to follow a tight relation such as a power law. The coupling between $\mathrm{PAH}$ and $\mathrm{H}_{2}$ implies that both are excited predominantly in PDRs, although they may not come from the exact same layers (at the same optical depths within the clouds).

A possible explanation for the large range of the constant $a$ may be caused by the different physical properties of the merger systems, e.g., a different abundance of warm molecular gas to PAHs. This would imply that any correlation between $\mathrm{H}_{2}$ and PAH emission based only on global measurements for a sample of different galaxies would be washed out, simply because the global measurement reflects only the static physical properties rather than the behavior of the $\mathrm{H}_{2}$ and $\mathrm{PAH}$ excitation within the galaxies. In principle, a possible contribution from mechanical heating through shocks may play a role as well on some local scales (e.g., for the center of NGC 6240, see Section 3.4).
However, this is likely not the dominant contribution in our galaxies since observations of extreme $\mathrm{H}_{2}$-luminous galaxies (Ogle et al. 2010; Nesvadba et al. 2010; Guillard et al. 2011) have shown that shock-excited galaxies typically have a larger $\mathrm{H}_{2} / \mathrm{PAH}$ ratio $^{14}$ than in our sample (e.g., the Stephans Quintet with $\mathrm{H}_{2}(\mathrm{~S}(0)-\mathrm{S}(3)) / \mathrm{PAH} 7.7 \mu \mathrm{m}>1$; Guillard et al. 2010).

\subsection{Local Variations versus Evolutionary Trends along the Toomre Sequence}

To investigate possible evolutionary effects on the gas and dust properties of major mergers we have studied several mid-IR features as a function of their merger stage, representing the early (NGC 4676, NGC 7592, NGC 6621-distinguishable parent disks and large tidal tails), mid (NGC 2623, NGC 6240, NGC 520-highly overlapping disks and/or double nuclei), and late (NGC 3921, NGC 7252 - single nucleus, elliptical-like light distribution) stages. For instance, a change in dust grain size, ionization stage, neutral to ionized gas fraction, or gas temperatures could be expected. To test such a possible scenario we study the following mid-IR diagnostics as a function of merger stage as shown in Figure 16: [Ne III]/[Ne II], the PAH interband strength ratios $11.3 / 17 \mu \mathrm{m}$ and $7.7 / 11 \mu \mathrm{m}$, the warm gas to $\mathrm{PAH}$ ratio $\mathrm{H}_{2}(\mathrm{~S} 0-\mathrm{S} 2) / \mathrm{PAH}$ as described in Section 3.3.4, the dust continuum ratios $30 / 15 \mu \mathrm{m}$ and $15 / 5.5 \mu \mathrm{m}$ as derived in Section 3.2.1, and the two warm $\mathrm{H}_{2}$ gas components. The results are presented for the entire merger systems as well as for the individual merger components. Furthermore we explored the radial profile and spatial size (derived from Sérsic profile fit) of several mid-IR features as a function of merger stage, such as the radial size ratio of $\mathrm{H}_{2} / \mathrm{IR}$ continuum, PAH $7.7 / 11.3 \mu \mathrm{m}$, and $\mathrm{H}_{2} / \mathrm{PAH}$. Our main conclusion is that the variety of mid-IR properties in different regions as shown in Figure 16 is significantly larger than any trend along the merger sequence using the global properties. This does not necessarily imply that an overall trend along the merger sequence does not exist, for which we find indications in the mid-IR continuum slope (Figure 16) and the relative radial size of the PAH interband emissions and the $\mathrm{H}_{2}$ emission as shown in Figure 17. However, a much larger sample would be required to statistically confirm such a trend in the mid-IR properties (see S. Stierwalt et al. 2011, in preparation, for nuclear mid-IR properties as function of merger stage).

Despite the small number of galaxies in our sample we find two galaxies that are outliers in one or more properties: NGC 6240 does not only show a significantly larger $\mathrm{H}_{2} / \mathrm{PAH}$ flux ratio (see Section 3.3.4), but also a larger PAH 11.3/17 $\mu \mathrm{m}$ flux ratio as well as a larger ratio of the radii $\mathrm{H}_{2} / \mathrm{IR}$ cont, $\mathrm{H}_{2} / \mathrm{PAH}$, and PAH 11.3/7.7 $\mu \mathrm{m}$ than most of the other galaxies in our sample. Previous observations of the center of this galaxy have revealed two nuclei with a separation of 1".8 (Fried \& Schulz 1983) and that the $\mathrm{H}_{2}$ emission peaks between the two nuclei and is thermally excited via slow shocks triggered by the collision of the two nuclei (van der Werf et al. 1993; Sugai et al. 1997; Tecza et al. 2000; Armus et al. 2006). This may explain the intense $\mathrm{H}_{2}$ emission in the very center of NGC 6240 (unresolved with Spitzer IRS; see Armus et al. 2006), but is likely not responsible for the correlation seen in the outer disk between $\mathrm{H}_{2}$ and PAH. Another galaxy, NGC 2623, is significantly larger

\footnotetext{
14 Note that in some studies the $\mathrm{H}_{2} / \mathrm{PAH}$ ratio is defined as $\mathrm{H}_{2}(\mathrm{~S}(0)-\mathrm{S}(3)) / \mathrm{PAH} 7.7 \mu \mathrm{m}$ which is for our sample typically a factor of $\sim 3$ larger than $\mathrm{H}_{2}(\mathrm{~S}(0)-\mathrm{S}(2)) / \mathrm{PAH}(6.2,7.7,11.3,17 \mu \mathrm{m})$, but the former ratio shows typically a larger variation on local scales and a less significant power law than the latter one.
} 

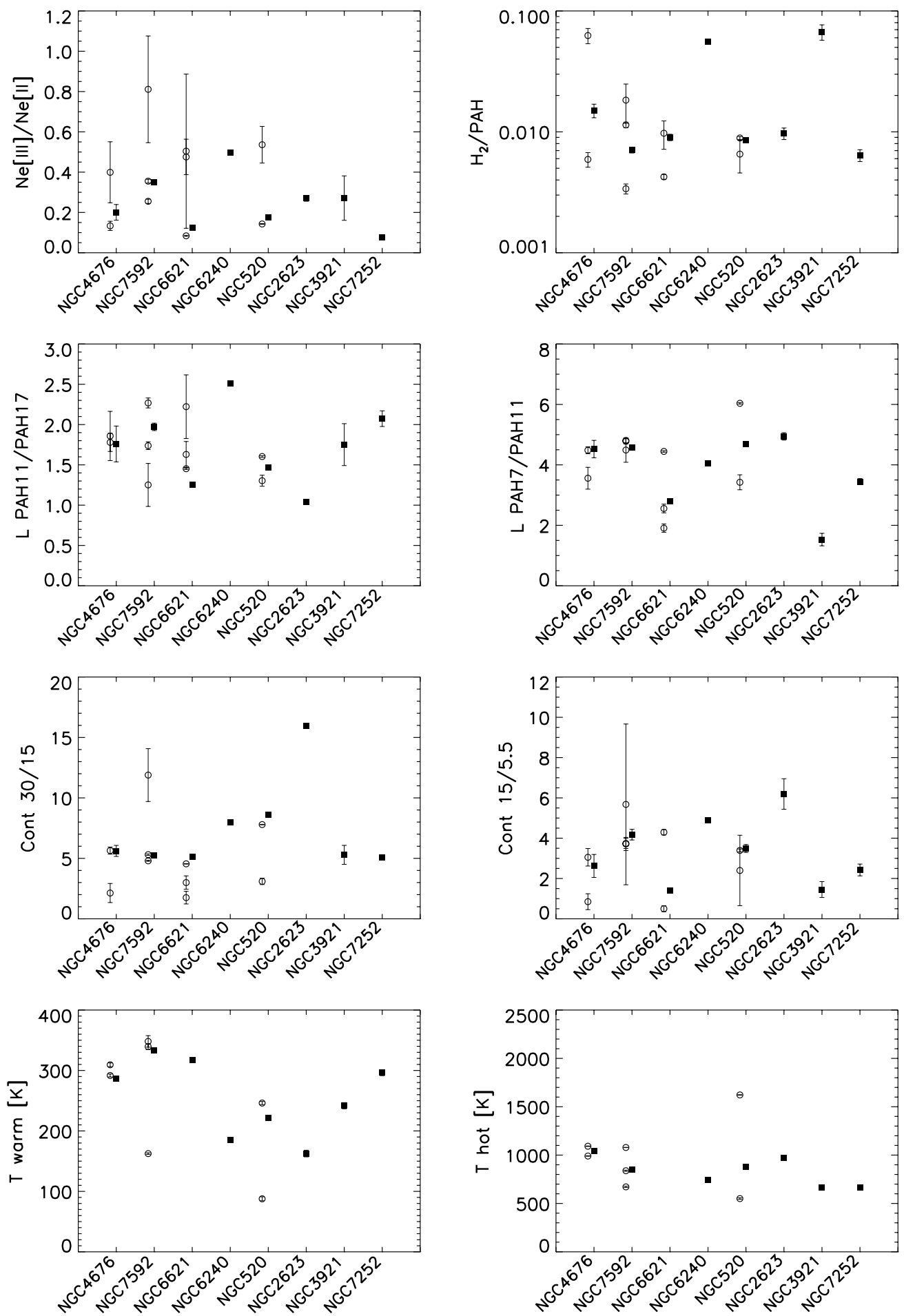

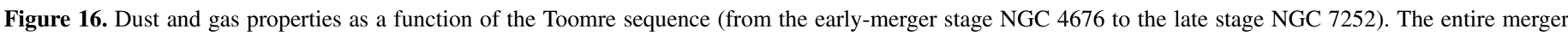

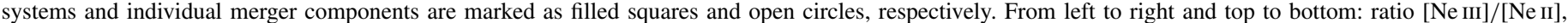

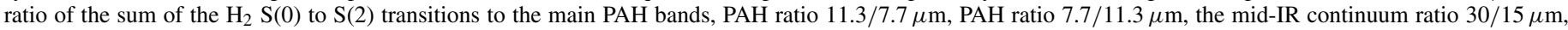
$15 / 5.5 \mu \mathrm{m}$, the "warm" $\mathrm{H}_{2}$ temperature, and "hot" $\mathrm{H}_{2}$ temperature component (see the text for details).

in the flux continuum ratio $(30 / 15 \mu \mathrm{m}=16.3)$ than the rest of the sample (mean $30 / 15 \mu \mathrm{m} \sim 6$ ), which may be due to the star formation intensity peak that occurs during a relatively short timescale $(<0.5 \mathrm{Gyr})$ at the final coalescence of the nuclei as shown in recent major merger simulations (Cox et al. 2008; Hopkins et al. 2009). Furthermore, this stage also seems to show a relative change in the radii of the mid-IR features, namely, a maximum in the $\mathrm{H}_{2}$ emission line radius to mid-IR continuum radius (at $5.5-15 \mu \mathrm{m}$ ) as well as in the $\mathrm{PAH} 11.3 \mu \mathrm{m}$ to $7.7 \mu \mathrm{m}$ line ratio radius.

\subsection{Multiwavelength View of the Interstellar Material in Early Merger Stages}

In this section, we discuss in more detail three "classical" major mergers, NGC 4676, NGC 7592, and NGC 6621, and compare their mid-IR properties with the distribution of the 

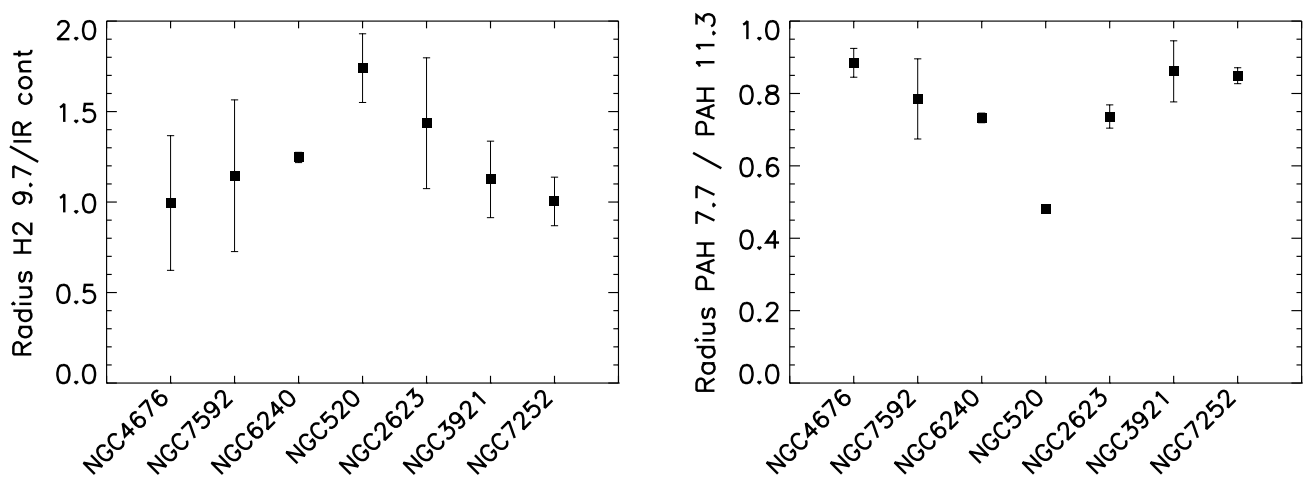

Figure 17. Relative radii of the major merger components as a function of the Toomre sequence (from the early merger stage NGC 4676 to the late stage NGC 7252 ). Left: the ratio of the strongest $\mathrm{H}_{2}$ emission line ( $\mathrm{S}(3)$ ) to the mid-IR continuum radius (at 5.5-15 $\mu \mathrm{m}$ ) as a function of merger stage. Right: the ratio of the PAH $7.7 \mu \mathrm{m}$ to $11.3 \mu \mathrm{m}$ line radius as a function of merger stage.

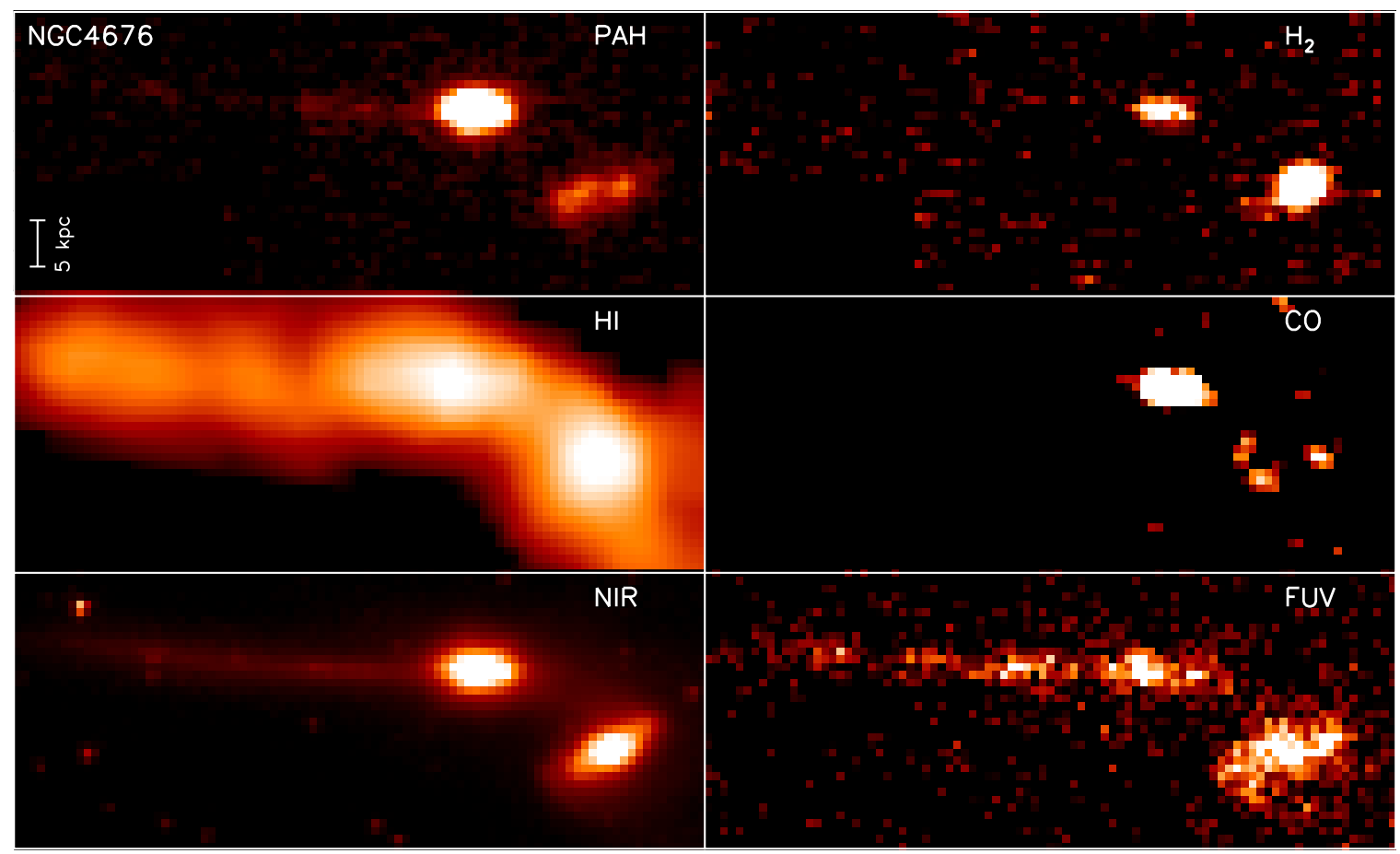

Figure 18. Multiwavelength view of NGC 4676, from left to right and top to bottom: the combined main PAH features, the warm molecular gas ( $\mathrm{H}_{2}$ emission), the cold atomic gas $(\mathrm{HI})$, the cold molecular gas (CO), the NIR (Spitzer IRAC $3.6 \mu \mathrm{m})$, and the FUV (GALEX). The north and east directions in each frame of this figure are the same as in the corresponding frame of Figure 2.

(A color version of this figure is available in the online journal.)

stellar (NIR and FUV), cold atomic (HI), and cold molecular (CO; see Yun \& Hibbard 2001) gas components. These three mergers are in particular interesting since they are caught in the early merger stages and have spatially resolved components, which allows us to compare in detail the physical properties between the two nuclei in each merger system.

\subsubsection{NGC 4676}

Nicknamed the "Mice," each of the two galaxies in NGC 4676 possesses a long tail as observed in the optical light. The two galaxies of this merger system have a projected separation distance of 36 ".8 $(\sim 17 \mathrm{kpc})$. In particular, the northern galaxy exhibits a long tidal tail with a projected extent of $48 \mathrm{kpc}$ (measured in the NIR), visible in the FUV, near-infrared, mid-IR, and H I emission (see Figure 18). Material is being exchanged between the two disks as indicated by the presence of a bright optical bridge between them. Stockton (1974) and Hibbard
(1995) found that the tails have widespread star formation which accounts for $16 \%$ of the total $\mathrm{H} \alpha$ emission and exhibit a high atomic gas content with on average $M_{\mathrm{HI}} / L_{R} \sim 0.4 M_{\odot} L_{\odot}^{-1}$, with the stellar tails exceeding the gaseous tails in extent.

Although both galaxies have roughly the same near-IR luminosities (as measured in the $H$ band), we find significant differences in the distribution of the mid-IR features. As shown in Figure 18, most of the PAH and $\mathrm{CO}$ emission (each $>90 \%$ ) originate from the northern galaxy, while the main $\mathrm{H}_{2}$ line emission $(>80 \%)$ is found in the southern galaxy. This finding may suggest a significantly different stellar population or distribution of molecular gas and young stars in the merger components. The northern component has a significantly smaller $[\mathrm{Ne}$ III] $/[\mathrm{Ne}$ II $]$ ratio $(0.13)$ and larger PAH 7.7/11.3 $\mu \mathrm{m}$ ratio (4.5) than the southern galaxy $(\sim 0.4$ and 3.5 , respectively), suggesting that the average stellar population is younger and the grains may be more ionized in the northern component. 


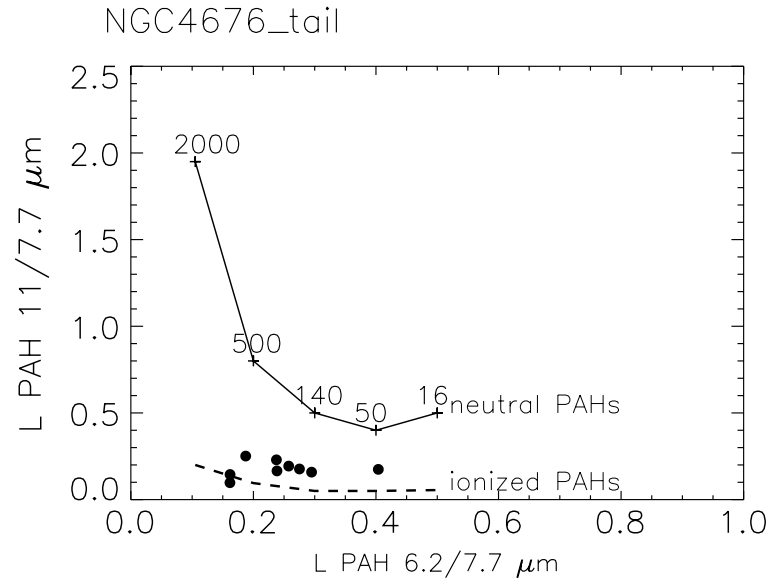

Figure 19. Variation of interband strength ratios of the PAH features in the tidal tail of NGC 4676 indicates emission from ionized PAHs. Same scales, markers, and labels as in Figure 7.

Interestingly, we find that the $\mathrm{H}_{2}$ temperature components ( $\sim 300 \mathrm{~K}$ for the warm and $\sim 1000 \mathrm{~K}$ for the hot component) are roughly the same in both galaxies. Thus, the strong $\mathrm{H}_{2}$ emission in the southern galaxy may be simply due to the presence of a larger amount of warm molecular gas $\left(\mathrm{H}_{2}\right.$ mass two times larger than in the northern galaxy).

Furthermore, we find PAH emission in the northern tidal tail, but no significant $\mathrm{H}_{2}$ emission. Figure 19 shows that the interband strength of the PAH 7.7/11 $\mu \mathrm{m}$ and 6.2/7.7 $\mu \mathrm{m}$ ratios of the tidal tail regions is all characterized by the ionized phase (using the model by Draine \& Li 2001). This is perhaps not surprising given the large number of young stars in the tidal tail as seen in the strong FUV emission (Figure 18) and $\mathrm{H} \alpha$ emission (Stockton 1974; Hibbard 1995; Hibbard \& van Gorkom 1996), demonstrating that the grain properties in interacting galaxies as traced by the mid-IR feature ratios can be affected over large scales in interacting systems.

\subsection{2. $N G C 7592$}

The two merging galaxies in this LIRG system $\left(L_{\mathrm{IR}} / L_{\odot}=\right.$ 10 ${ }^{11.33}$; Sanders et al. 2003) have a projected separation of $14^{\prime \prime}$ ( $\sim 7 \mathrm{kpc}$ ), a NIR luminosity ratio of $\sim 1: 1$, and show tidal tails in the NIR light. Figure 20 highlights the details of this system at multiple wavelengths. Like NGC 4676, the PAH and $\mathrm{H}_{2}$ emissions have a different distribution, with the PAH emission primarily originating from the eastern starbursting component and the $\mathrm{H}_{2}$ from the western component, which has a Seyfert 2 nucleus (Laine et al. 2003).

We find an additional concentration in the overlap region between the two nuclei (with an offset of $\sim 12^{\prime \prime}$ toward south) in the NIR light, FUV light, H I emission, and in several mid-IR features (main PAH features, [Ne II], and [Ne III]). No significant $\mathrm{H}_{2}$ emission is found at this position. The $\mathrm{PAH}$ interband strength and $[\mathrm{Ne}$ III $] /[\mathrm{Ne}$ II $]$ ratios indicate that most of the emission from this intermediate region originates from ionized gas (see Figure 7), which is likely due to star formation as we find a strong emission peak in the FUV and $\mathrm{H} \alpha$ (Laine et al. 2003) at this position as well.

\subsubsection{NGC 6621}

The two merging components of NGC 6621 have a projected separation of $41^{\prime \prime}(\sim 17 \mathrm{kpc})$. Unlike the two previous discussed

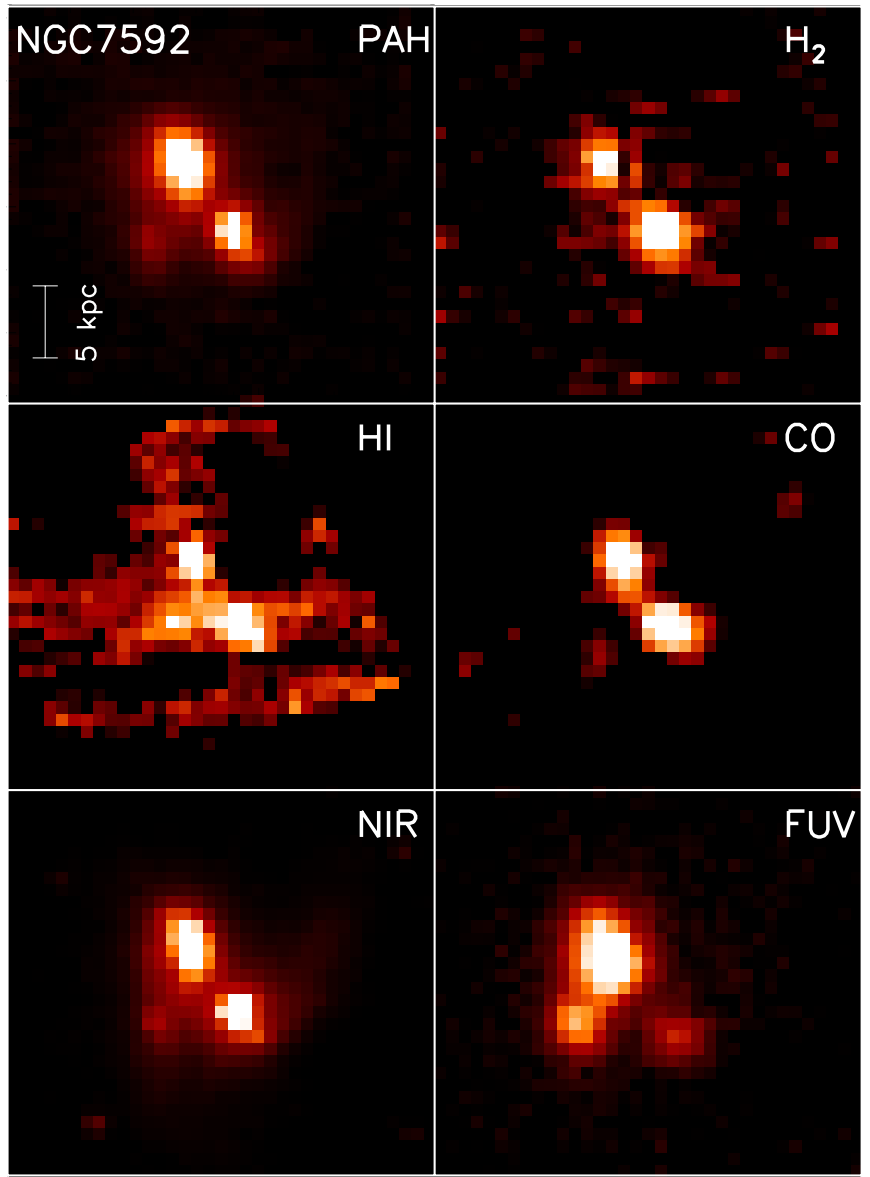

Figure 20. Multiwavelength view of NGC 7592, from left to right and top to bottom: the combined PAH features, the warm molecular gas $\left(\mathrm{H}_{2}\right.$ emission), the cold atomic gas (HI), the cold molecular gas (CO), the NIR (Spitzer IRAC $3.6 \mu \mathrm{m})$, and the FUV (GALEX). The north and east directions in each frame of this figure are the same as in the corresponding frame of Figure 2.

(A color version of this figure is available in the online journal.)

major mergers, NGC 4676 and NGC 7592, which have a NIR light ratio of $1: 1$, the two components of this system have a ratio of $\sim 1: 2$. However, this ratio changes dramatically in the light of different wavelengths. The mid-IR light of the smaller component, as given by our IRS continuum measurement, is at least two orders smaller than the larger component (nearly all of the mid-IR emission in NGC 6621 is coming from only one of the two nuclei). As shown in Figure 21, we find no significant $\mathrm{FUV}, \mathrm{H}_{2}, \mathrm{CO}$, and $\mathrm{H} \mathrm{I}$ emission at the position of the smaller galaxy, and only very faint emission from $\mathrm{PAH}$ features. Instead, we find significant emission originating from a peak in the overlap region between the two merging galaxies. The interband PAH strength ratios of this peak (see Figure 7) indicate that the PAH component may originate partially from neutral gas layers. However, we also find significant $\mathrm{H}_{2}$ and FUV emission in this overlap region, suggesting a burst of star formation here.

The distribution of the cold molecular gas, as observed in the CO emission line (Iono et al. 2005), is not co-spatial to the stellar (NIR), warm gas $\left(\mathrm{H}_{2}\right)$, and PAH distribution: besides the $\mathrm{CO}$ emission peak of the main galaxy, we find an additional CO concentration ( $\sim 30 \%$ of the total CO emission) between the main galaxy and the overlap region as seen in the FUV and NIR light, and mid-IR features. This significant offset suggests 


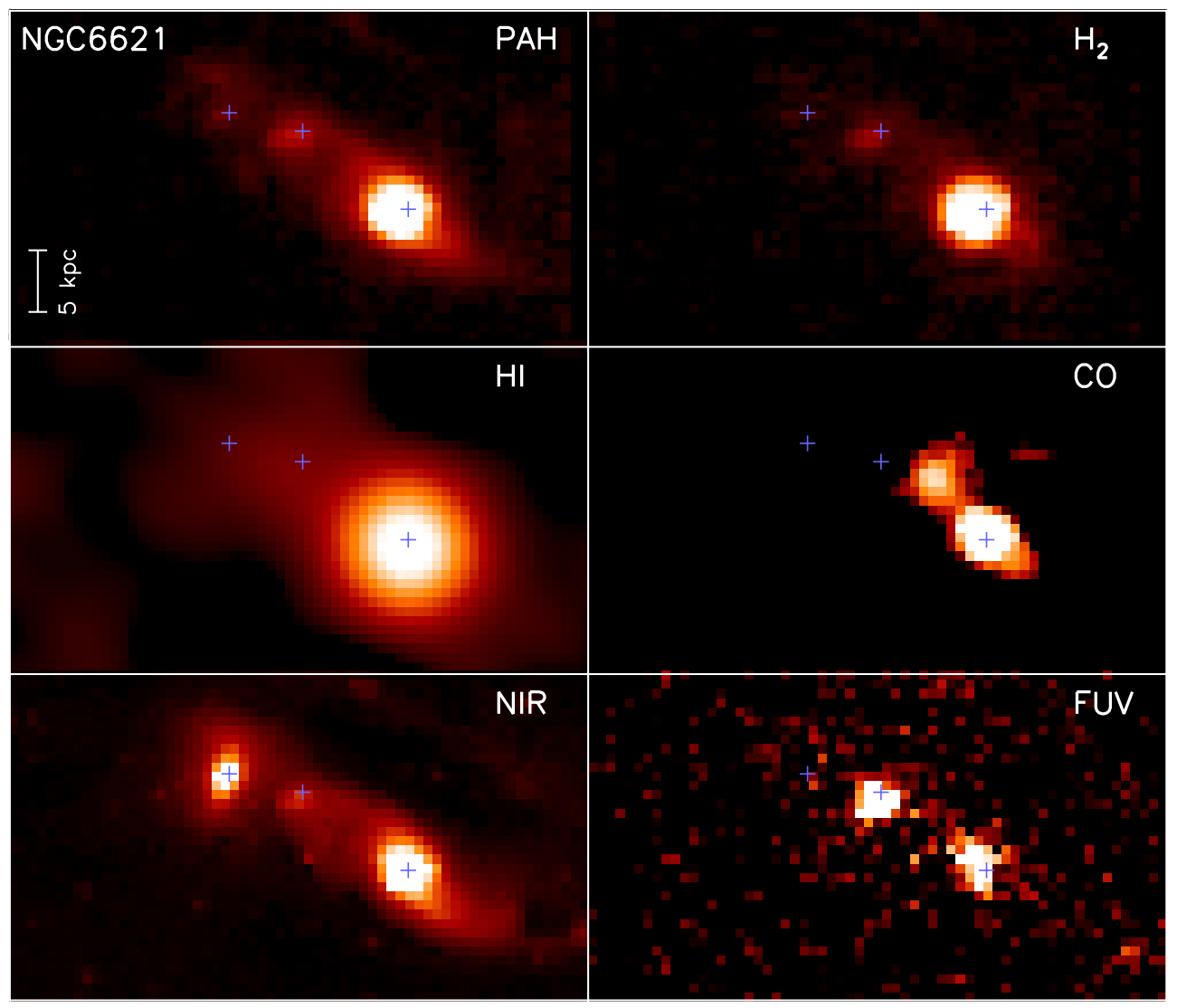

Figure 21. Multiwavelength view of NGC6621, from left to right and top to bottom: the combined PAH features, the warm molecular gas ( $\mathrm{H}_{2}$ emission), the cold atomic gas (HI), the cold molecular gas (CO), the NIR (Spitzer IRAC $3.6 \mu \mathrm{m}$ ), and the FUV (GALEX). The centers of the two galaxies (as seen in the NIR) and the star formation peak in between are indicated with a blue plus marker. The north and east directions in each frame of this figure are the same as in the corresponding frame of Figure 2.

(A color version of this figure is available in the online journal.)

a large reservoir of cold molecular gas with no signs of star formation in the mid-IR features.

\section{CONCLUSIONS}

We analyzed the spatial distribution and properties of the main mid-IR features observed with Spitzer/IRS for eight major merger systems of the Toomre sequence. Maps of the key diagnostic features of the physical conditions in these interacting starburst galaxies are presented - such as PAH ratios, ionized emission line regions, the warm molecular gas $\left(\mathrm{H}_{2}\right)$ distribution, and temperatures. Moreover, we have compared the mid-IR features to the cold atomic and molecular gas distribution to obtain a comprehensive view about the variation and evolution of the interstellar material phase space in major mergers. The main results are summarized in the following.

1. The range and distribution of the global PAH interband strength and $[\mathrm{Ne} \mathrm{III}] /[\mathrm{Ne} \mathrm{II}]$ ratios of major mergers are very similar to normal star-forming galaxies. However, regarding the distribution of these properties over all spatial scales within the galaxies, we find significantly different PAH populations (defined by their ionization state and grain size) among the merger systems in our sample.

2. The cold molecular gas component as observed in the $\mathrm{CO}$ emission line does not usually correlate with the warm gas in early merger systems, leading to large spatial variations in the ratio of cold-to-warm molecular gas. In particular, the cold molecular gas distribution in NGC 6621 shows a significant offset not only from the warm gas component, but also from the stellar (NIR) and dust distribution (PAH, mid-IR continuum).

3. Two merger systems (NGC 7592 and NGC 6621) show evidence for star formation in overlap regions between the nuclei, namely, strong PAH, [Ne II], [Ne III], and warm gas $\left(\mathrm{H}_{2}\right)$ emission, but no apparent corresponding $\mathrm{CO}(J=1-0)$ emission.

4. The majority of galaxies in our merging sequence sample have similar global $\mathrm{H}_{2}$-to-PAH flux ratios as normal star-forming galaxies with $\mathrm{H}_{2}$ temperatures ranging from $\sim 90 \mathrm{~K}$ to $1100 \mathrm{~K}$. Three starburst galaxies in our sample (NGC 4676 South, NGC 6240, and NGC 3921) have a factor of 10 larger ratios, clearly separated from the rest of our sample and normal star-forming galaxies, and even larger than what it is in most AGN galaxies in the SINGS sample (Roussel et al. 2007). However, regarding the different apertures (locations) within a merger, we find an intrinsic relation between $\mathrm{H}_{2}$ and PAH flux as given by a power law with a very similar exponent $(0.61 \pm 0.05)$ for all galaxies but with a constant that can vary up to a factor of 10 between different galaxies. Such a tight correlation between PAH and $\mathrm{H}_{2}$ over all spatial locations suggests a common dominant excitation mechanism for $\mathrm{H}_{2}$ emission in major mergers, which is most likely absorption of UV photons in PDRs rather than shock excitation, since PAHs are known to arise predominantly from these regions. 
5. The minimum of the $11.3 / 7.7 \mu \mathrm{m}$ PAH interband strength ratio is typically located in the nuclei of galaxies, suggesting a larger ionization state of the ISM toward the center, as expected from central starbursts or low-luminosity AGNs (Seyfert, LINER). The [Ne III/[Ne II] ratio increases with distance from the nucleus, seemingly conflicting the expectation of a decrease of the hardening of the radiation field with distance from the center. A possible explanation for a suppressed [Ne III]/[Ne II] ratio in the center is either a larger contribution of the old stellar population in the nuclei or a fraction of the most massive stars are hidden in ultracompact $\mathrm{H}$ II regions due to high dust densities in the nuclear regions. Thus, the $[\mathrm{Ne}$ III/[Ne II] ratio is likely more sensitive to the average stellar age and/or gas density rather than a hardening of the field, suggesting that the $11.3 / 7.7 \mu \mathrm{m}$ PAH ratio is a better diagnostic of the ionization state in all merger components, as seen in the center and outskirts of galaxies, in overlapping regions between merger components, and in tidal tails.

6. Variations of the internal (local) physical conditions within a merger are much larger than the systematic (global) variations along the Toomre sequence. Note that this does not necessarily exclude the possibility of an evolutionary trend, for which we find indications in the mid-IR continuum slope and the relative radial size of the PAH interband emissions and the $\mathrm{H}_{2}$ emission.

Support for this work was provided by NASA through a grant issued by the Spitzer Science Center and the Jet Propulsion Laboratory. This research has made use of the NASA/IPAC Extragalactic Database, which is operated by the Jet Propulsion Laboratory, California Institute of Technology, under contract with the National Aeronautics and Space Administration. We thank the referee for a careful review and valued suggestions, and Pierre Guillard for a useful discussion.

\section{REFERENCES}

Allamandola, L. J., Hudgins, D. M., \& Sandford, S. A. 1999, ApJ, 511, L115

Alonso-Herrero, A., Pereira-Santaella, M., Rieke, G. H., et al. 2010, Adv. Space Res., 45, 99

Armus, L., Bernard-Salas, J., Spoon, H. W. W., et al. 2006, ApJ, 640, 204

Armus, L., Charmandaris, V., Bernard-Salas, J., et al. 2007, ApJ, 656, 148

Armus, L., Charmandaris, V., Spoon, H. W. W., et al. 2004, ApJS, 154, 178

Armus, L., Heckman, T., \& Miley, G. 1987, AJ, 94, 831

Barnes, J. E., \& Hernquist, L. 1992, ARA\&A, 30, 705

Beirão, P., Brandl, B. R., Appleton, P. N., et al. 2008, ApJ, 676, 304

Bernard-Salas, J., Spoon, H. W. W., Charmandaris, V., et al. 2009, ApJS, 184, 230

Blain, A. W., Smail, I., Ivison, R. J., Kneib, J.-P., \& Frayer, D. T. 2002, Phys. Rep., 369, 111

Brandl, B. R., Bernard-Salas, J., Spoon, H. W. W., et al. 2006, ApJ, 653, 1129

Casoli, F., Dupraz, C., Combes, F., \& Kazes, I. 1991, A\&A, 251, 1

Chapman, S. C., Blain, A. W., Smail, I., \& Ivison, R. J. 2005, ApJ, 622, 772

Charmandaris, V., Laurent, O., Le Floc'h, E., et al. 2002, A\&A, 391, 429

Chitre, A., \& Jog, C. J. 2002, A\&A, 388, 407

Côté, P., Ferrarese, L., Jordán, A., et al. 2007, ApJ, 671, 1456

Cox, T. J., Jonsson, P., Somerville, R. S., Primack, J. R., \& Dekel, A. 2008, MNRAS, 384, 386

Devost, D., Brandl, B. R., Armus, L., et al. 2004, ApJS, 154, 242

Diamond-Stanic, A. M., \& Rieke, G. H. 2010, ApJ, 724, 140

Díaz-Santos, T., Alonso-Herrero, A., Colina, L., et al. 2010a, ApJ, 711, 328

Díaz-Santos, T., Charmandaris, V., Armus, L., et al. 2010b, ApJ, 723, 993

Díaz-Santos, T., Charmandaris, V., Armus, L., et al. 2011, ApJ, 741, 32

Draine, B. T., \& Li, A. 2001, ApJ, 551, 807
Draine, B. T., Roberge, W. G., \& Dalgarno, A. 1983, ApJ, 264, 485

Ferrarese, L., Côté, P., Jordán, A., et al. 2006, ApJS, 164, 334

Fried, J. W., \& Schulz, H. 1983, A\&A, 118, 166

Galliano, F., Madden, S. C., Tielens, A. G. G. M., Peeters, E., \& Jones, A. P. 2008, ApJ, 679, 310

Giveon, U., Sternberg, A., Lutz, D., Feuchtgruber, H., \& Pauldrach, A. W. A. 2002, ApJ, 566, 880

Guillard, P., Boulanger, F., Cluver, M. E., et al. 2010, A\&A, 518, A59

Guillard, P., et al. 2011, ApJ, in press

Haan, S., Surace, J. A., Armus, L., et al. 2011, AJ, 141, 100

Habart, E., Boulanger, F., Verstraete, L., et al. 2003, A\&A, 397, 623

Hao, L., Wu, Y., Charmandaris, V., et al. 2009, ApJ, 704, 1159

Heckman, T. M., Armus, L., \& Miley, G. K. 1990, ApJS, 74, 833

Hibbard, J. E. 1995, PhD thesis, Columbia Univ.

Hibbard, J. E., \& van Gorkom, J. H. 1996, AJ, 111, 655

Higdon, S. J. U., Armus, L., Higdon, J. L., Soifer, B. T., \& Spoon, H. W. W. 2006, ApJ, 648, 323

Hollenbach, D. J., \& Tielens, A. G. G. M. 1997, ARA\&A, 35, 179

Hopkins, P. F., Cox, T. J., Dutta, S. N., et al. 2009, ApJS, 181, 135

Houck, J. R., Roellig, T. L., van Cleve, J., et al. 2004, ApJS, 154, 18

Houck, J. R., Schneider, D. P., Danielson, G. E., et al. 1985, ApJ, 290, L5

Iono, D., Yun, M. S., \& Ho, P. T. P. 2005, ApJS, 158, 1

Joseph, R. D., \& Wright, G. S. 1985, MNRAS, 214, 87

Kormendy, J., Fisher, D. B., Cornell, M. E., \& Bender, R. 2009, ApJS, 182, 216

Laine, S., Appleton, P. N., Gottesman, S. T., Ashby, M. L. N., \& Garland, C. A. 2010, AJ, 140, 753

Laine, S., van der Marel, R. P., Rossa, J., et al. 2003, AJ, 126, 2717

Lepp, S., \& McCray, R. 1983, ApJ, 269, 560

Maloney, P. R., Hollenbach, D. J., \& Tielens, A. G. G. M. 1996, ApJ, 466, 561

Mihos, J. C., \& Hernquist, L. 1994, ApJ, 437, L47

Murphy, T. W., Jr., Armus, L., Matthews, K., et al. 1996, AJ, 111, 1025

Nesvadba, N. P. H., Boulanger, F., Salomé, P., et al. 2010, A\&A, 521, A65

O’Dowd, M. J., Schiminovich, D., Johnson, B. D., et al. 2009, ApJ, 705, 885

Ogle, P., Boulanger, F., Guillard, P., et al. 2010, ApJ, 724, 1193

Peeters, E., Spoon, H. W. W., \& Tielens, A. G. G. M. 2004, ApJ, 613, 986

Pereira-Santaella, M., Alonso-Herrero, A., Rieke, G. H., et al. 2010, ApJS, 188, 447

Rand, R. J., Wood, K., \& Benjamin, R. A. 2008, ApJ, 680, 263

Rigby, J. R., \& Rieke, G. H. 2004, ApJ, 606, 237

Rigopoulou, D., Kunze, D., Lutz, D., Genzel, R., \& Moorwood, A. F. M. 2002, A\&A, 389, 374

Rossa, J., Laine, S., van der Marel, R. P., et al. 2007, AJ, 134, 2124

Roussel, H., Helou, G., Hollenbach, D. J., et al. 2007, ApJ, 669, 959

Sales, D. A., Pastoriza, M. G., \& Riffel, R. 2010, ApJ, 725, 605

Sanders, D. B., Mazzarella, J. M., Kim, D.-C., Surace, J. A., \& Soifer, B. T. 2003, AJ, 126, 1607

Sanders, D. B., Scoville, N. Z., Young, J. S., et al. 1986, ApJ, 305, L45

Sanders, D. B., Soifer, B. T., Elias, J. H., et al. 1988, ApJ, 325, 74

Schweizer, F. 1982, ApJ, 252, 455

Schweizer, F., Miller, B. W., Whitmore, B. C., \& Fall, S. M. 1996, AJ, 112, 1839

Scoville, N. Z., Sargent, A. I., Sanders, D. B., \& Soifer, B. T. 1991, ApJ, 366, L5

Shull, J. M., \& Hollenbach, D. J. 1978, ApJ, 220, 525

Smith, J. D. T., Armus, L., Dale, D. A., et al. 2007, PASP, 119, 1133

Smith, J. D. T., Draine, B. T., Dale, D. A., et al. 2007, ApJ, 656, 770

Snijders, L., Kewley, L. J., \& van der Werf, P. P. 2007, ApJ, 669, 269

Soifer, B. T., Neugebauer, G., Helou, G., et al. 1984, ApJ, 283, L1

Steidel, C. C., Giavalisco, M., Pettini, M., Dickinson, M., \& Adelberger, K. L. 1996, ApJ, 462, L17

Stockton, A. 1974, ApJ, 187, 219

Sugai, H., Malkan, M. A., Ward, M. J., Davies, R. I., \& McLean, I. S. 1997, ApJ, 481,186

Surace, J. A., Sanders, D. B., \& Mazzarella, J. M. 2004, AJ, 127, 3235

Tecza, M., Genzel, R., Tacconi, L. J., et al. 2000, ApJ, 537, 178

Thornley, M. D., Schreiber, N. M. F., Lutz, D., et al. 2000, ApJ, 539, 641

Toomre, A. 1977, in Evolution of Galaxies and Stellar Populations, ed. B. M.

Tinsley \& R. B. Larson (New Haven, CT: Yale University Observatory), 401 Toomre, A., \& Toomre, J. 1972, ApJ, 178, 623

van der Werf, P. P., Genzel, R., Krabbe, A., et al. 1993, ApJ, 405, 522

Veilleux, S., Kim, D.-C., Rupke, D. S. N., et al. 2009, ApJ, 701, 587

Velusamy, T., \& Langer, W. D. 2008, AJ, 136, 602

Yun, M. S., \& Hibbard, J. E. 2001, ApJ, 550, 104

Zakamska, N. L. 2010, Nature, 465, 60 